\title{
COMPUTATIONAL MODELS OF CERTAIN HYPERSPACES OF QUASI-METRIC SPACES
}

\author{
MAHDI ALI-AKBARI AND MASSOUD POURMAHDIAN
}

Department of Mathematics, Semnan University, P.O. Box 35195-363, Semnan, Iran and School of Mathematics, Institute for Research in Fundamental Sciences (IPM), Tehran, Iran.

e-mail address: m_aliakbari@aut.ac.ir

School of Mathematics and Computer Science, Amirkabir University of Technology, Tehran, Iran and School of Mathematics, Institute for Research in Fundamental Sciences (IPM), Tehran, Iran. e-mail address: pourmahd@ipm.ir

\begin{abstract}
In this paper, for a given sequentially Yoneda-complete $T_{1}$ quasi-metric space $(X, d)$, the domain theoretic models of the hyperspace $\mathcal{K}_{0}(X)$ of nonempty compact subsets of $(X, d)$ are studied. To this end, the $\omega$-Plotkin domain of the space of formal balls $\mathbf{B} X$, denoted by $\mathbf{C B} X$ is considered. This domain is given as the chain completion of the set of all finite subsets of $\mathbf{B} X$ with respect to the Egli-Milner relation. Further, a map $\phi: \mathcal{K}_{0}(X) \rightarrow \mathbf{C B} X$ is established and proved that it is an embedding whenever $\mathcal{K}_{0}(X)$ is equipped with the Vietoris topology and respectively $\mathbf{C B} X$ with the Scott topology. Moreover, if any compact subset of $(X, d)$ is $d^{-1}$-precompact, $\phi$ is an embedding with respect to the topology of Hausdorff quasi-metric $H_{d}$ on $\mathcal{K}_{0}(X)$. Therefore, it is concluded that $(\mathbf{C B} X, \sqsubseteq, \phi)$ is an $\omega$-computational model for the hyperspace $\mathcal{K}_{0}(X)$ endowed with the Vietoris and respectively the Hausdorff topology.

Next, an algebraic sequentially Yoneda-complete quasi-metric $D$ on $\mathbf{C B} X$ is introduced in such a way that the specialization order $\sqsubseteq_{D}$ is equivalent to the usual partial order of $\mathbf{C B} X$ and, furthermore, $\phi:\left(\mathcal{K}_{0}(X), H_{d}\right) \rightarrow(\mathbf{C B} X, D)$ is an isometry. This shows that $(\mathbf{C B} X, \sqsubseteq, \phi, D)$ is a quantitative $\omega$-computational model for $\left(\mathcal{K}_{0}(X), H_{d}\right)$.
\end{abstract}

\section{INTRODUCTION}

In this paper, we further continue a project carried out to investigate connections between domain theory and quasi-metric spaces [AHPR09]. Here, we provide some domain theoretic (computational) models for the hyperspace of nonempty compact subsets of quasi-metric spaces. On one hand, the recent applications of quasi-metric spaces in different subjects of computer science, e.g. denotational semantics of programming languages, complexity and dual-complexity spaces and complexity distances between algorithms ([RV08, RSV09, GRS08, RSV03, RS99, RRV08]) and, on the other hand, a new insight of the domain

1998 ACM Subject Classification: F.1.1.

Key words and phrases: Quasi-metric spaces, Yoneda and Smyth completeness, hyperspace of non-empty compact subsets, $(\omega$-)computational models, $\omega$-Plotkin domain.

The authors are partially supported by IPM, grants No. 89540064 and No. 89030120. 
theoretic point of view into the theory of hyperspaces and its new applications within mathematics, e.g. discrete dynamical systems, measure and integration theory (Eda95a, Eda97, Eda95b]), motivate establishing computational models of these structures.

Finding a domain theoretic (computational) model for a topological space $(X, \tau)$ amounts to providing a suitable partially ordered set $(P, \sqsubseteq)$ together with a topological embedding $\phi$ from $(X, \tau)$ to $(P, \sqsubseteq)$ endowed with the Scott topology, denoted by $\sigma$. This is a variant of a fundamental problem in domain theory, called the maximal point space problem, which demands a homeomorphism between $(X, \tau)$ and the space of maximal point of $(P, \sqsubseteq)$. The study of computational models for various type of topological spaces goes back to the works of Edalat and respectively Blanck [Eda95a, Bla00, Bla97]. Later, the maximal point space problem was explicitly formulated and became a subject of intensive investigations by many authors [Law97, BL08, Mar98, Rut98. Some special cases of this problem have satisfactory solutions [AHP09, KKW04].

The domain theoretic construction $\mathbf{B} X$ of the space of formal balls, introduced by Edalat and Heckmann, provides a concrete (computational) model for a metric space $(X, d)$ [EH98. The importance of this construction is that, first of all, it connects some metric properties of $(X, d)$ to the order theoretic properties of $\mathbf{B} X$. Secondly, it ties the above notion of computational model to the notion of computability for metric space $(X, d)$ ES99, Law98. The notion of formal balls is also defined in the same way for a quasi-metric space $(X, d)$ and the order theoretic properties of $\mathbf{B} X$ are tightly connected to the topological properties of $(X, d)$ AHPR09, RV09, RV10. In particular, for a $T_{1}$ quasi-metric space $(X, d)$ is sequentially Yoneda-complete if and only if $\mathbf{B} X$ is a directed complete partially ordered set.

Edalat and Heckmann also constructed the Plotkin powerdomain $\mathcal{P} \mathbf{B} X$ of the space of formal balls of a metric space $(X, d)$ and showed that there is a one-to-one correspondence between the nonempty compact subsets of $(X, d)$ and the maximal elements of the Plotkin powerdomain of $\mathbf{B} X$. As an application, a domain theoretic proof was given for a classical result of Hutchinson ([Hut85]) which states that if $(X, d)$ is complete, then any hyperbolic iterated function system has a unique non-empty compact attractor. It can be shown that this construction is a computational model for the hyperspace of nonempty compact subsets of $X$, denoted by $\mathcal{K}_{0}(X)$, with the Vietoris or equivalently the Hausdorff topology. This fact was also proved in a different way by Martin in [Mar04. His interesting idea is based on the existence of a certain measurement, called Lebesgue measurement, on any domain $D$ which models the metric space $(X, d)$. Subsequently, Liang and Kou in LK04 generalized these results to continuous dcpo's which have the Lawson condition, i.e. the Lawson and Scott topologies coincide on the space of maximal points. Indeed, under the Lawson condition, it is proved that there is a homeomorphism between the space of nonempty compact subsets of maximal points of a continuous dcpo $D$ endowed with the Vietoris topology and the space of maximal points of Plotkin powerdomain $D$ equipped with the induced Scott topology. More recently, in another line of research, Berger et al. ([Ber10]) showed that for any $T_{1}$ topological space which is represented by an $\omega$-domain $D$, the hyperspace of its nonempty compact subsets can be represented by the Plotkin powerdomain $\mathcal{P} D$ of domain $D$. This result was made possible by a theorem of Smyth (Smy83) which states that for any $\omega$-continuous dcpo $D$ the space $(\mathcal{P} D, \sigma)$ is homeomorphic to the space of $D$-lenses, i.e. nonempty compact subsets of domain $D$ which are intersection of a closed set and a saturated set, endowed with the Vietoris topology. 
In the present work, we study computational models of the hyperspace $\mathcal{K}_{0}(X)$ of a $T_{1}$ quasi-metric space $(X, d)$ equipped with the Vietoris and respectively the Hausdorff topology, proving that whenever $(X, d)$ satisfies certain completeness properties, e.g. Yoneda and respectively Smyth completeness, this space has a computational model. It is worth mentioning that the space of formal balls of a quasi-metric space does not generally satisfy the Lawson nor countable based conditions. Therefore, the results of Liang and Kou [LK04] and Berger et al. Ber10 do not apply to the present context. Also, unlike the metric case, there is no natural candidate for a measurement on the space of formal balls of a quasimetric space and hence the method used by Martin in Mar04 cannot be applied here either.

Edalat and Heckmann used the Plotkin powerdomain $\mathcal{P} \mathbf{B} X$ given as the ideal completion of the abstract basis of finite subset of $\mathbf{B} X, \mathcal{P}_{\text {fin }} \mathbf{B} X$, with respect to the Egli-milner relation, $\prec_{E M}$, to present a computational model of $\mathcal{K}_{0}(X)$, for every metric space $(X, d)$. To this end, they employed the symmetry axiom of metric $d$, to get a key fact that any maximal ideal has a cofinal $\omega$-chain. In the case of quasi-metric spaces, the Plotkin powerdomain $\mathcal{P} \mathbf{B} X$ can also be defined, though, the lack of symmetry for the quasi-metric $(X, d)$ prevents us from finding cofinal $\omega$-chains in maximal ideals. That is why we prefer to work directly with the $\omega$-chains and this leads us to the chain-completion construction instead.

So, for a $T_{1}$ quasi-metric space $(X, d)$, we consider the space $\mathbf{B} X$ of formal balls and let $\mathbf{C B} X$ be the chain completion of $\left(\mathcal{P}_{f i n} \mathbf{B} X, \prec_{E M}\right)$. This construction is called the $\omega$-Plotkin domain. By the general construction of chain completion, $\mathbf{C B} X$ is a continuous $\omega$-dcpo, i.e. a continuous poset in which every $\omega$-chain has a least upper bound. Now, to achieve our purpose in finding a computational model, we define a one-to-one map $\phi: \mathcal{K}_{0}(X) \rightarrow \mathbf{C B} X$, which is an embedding if we consider the Vietoris topology on $\mathcal{K}_{0}(X)$ and assume that $(X, d)$ is a sequentially Yoneda-complete $T_{1}$ quasi-metric space. Moreover, $\phi$ is an embedding with respect to the topology of the Hausdorff quasi-metric $H_{d}$ on $\mathcal{K}_{0}(X)$ if any compact subset of $X$ is $d^{-1}$-precompact. Therefore, $(\mathbf{C B} X, \phi)$ serves as an $\omega$-computational model for $\mathcal{K}_{0}(X)$ endowed with the mentioned topologies. Although it is not known whether $\mathbf{C B} X$ is a dcpo and therefore a computational model of $\mathcal{K}_{0}(X)$, nevertheless, thanks to Fact 1.1 and Theorem 2.5, the ideal completion of $\mathbf{C B} X$ gives a computational model for $\mathcal{K}_{0}(X)$.

In section 5, we take another well-known notion of computational model, called the quantitative $\omega$-computational model. This is an $\omega$-computational model $(P, \sqsubseteq, \phi)$ carrying an additional quasi-metric $D$ such that $\phi$ is an isometry from $(X, d)$ into $(P, D)$ together with some extra conditions which capture the order structure of $(P, \sqsubseteq)$ (Definition 4.1). A modified version of this notion can be found in RV09, Rut98, Sch03, Was06. We prove that in fact $\mathbf{C B} X$ is a quantitative $\omega$-computational model for $\left(\mathcal{K}_{0}(X), H_{d}\right)$, by constructing a quasi-metric $D$ on $\mathbf{C B} X$. To this end, we consider a quasi-metric $q$ defined by Romaguera and Valero ([RV09]) on $\mathbf{B} X$. The primary reason to choose this quasi-metric on $\mathbf{B} X$ is that $(\mathbf{B} X, q)$ is a quantitative computational model for $(X, d)$. Therefore, its specialization order $\sqsubseteq_{q}$ is equivalent to the partial order of $\mathbf{B} X$. Consequently, one could naturally extend $q$ to the Hausdorff quasi-metric $H_{q}$ on $\mathcal{P}_{\text {fin }} \mathbf{B} X$ of the finite subsets of $\mathbf{B} X$, whose main property is that it induces the Egli-Milner relation on $\mathcal{P}_{\text {fin }} \mathbf{B} X$. Subsequently, the quasi-metric $H_{q}$ can be lifted up to a quasi-metric $D$ on $\mathbf{C B} X$ in such a way that the ordered structures $\left(\mathbf{C B} X, \sqsubseteq_{D}\right)$ and $(\mathbf{C B} X, \sqsubseteq)$ coincide. Once $D$ is established one can show that $(\mathbf{C B} X, D)$ is a Yoneda-complete space and in fact Yoneda-completion of $\left(\mathcal{P}_{f i n} \mathbf{B} X, H_{q}\right)$. This makes $(\mathbf{C B} X, \sqsubseteq, \phi, D)$ a quantitative $\omega$-computational model for $\left(\mathcal{K}_{0}(X), H_{d}\right)$. 
We, finally, conclude this paper by comparing the Plotkin powerdomain and the $\omega$ Plotkin domain constructions. We prove that if $(X, d)$ is either, Smyth-complete and all of its compact subsets are $d^{-1}$-precompact, or an $\omega$-algebraic Yoneda-complete space, then the Plotkin Powerdomain $\mathcal{P} \mathbf{B} X$ is order-isomorphic to $\mathbf{C B} X$.

\section{Preliminaries}

We assume the reader is familiar with the basic definitions and facts about domain theory which can be found in ( AJ94, $\mathrm{GHK}^{+} 03$ ), though, we briefly explain some of the definitions and facts which are more crucial in this note.

Let $(P, \sqsubseteq)$ be a partially ordered set (abbr. by poset). The binary relation $\prec$ is called an auxiliary relation on the poset $(P, \sqsubseteq)$ if (1) $p \prec p$ implies $p \sqsubseteq p,(2) p \sqsubseteq s \prec r \sqsubseteq q$ implies $p \prec q$ and (3) satisfies the interpolation property, i.e. for any finite subset $M$ of $P$ and $p \in P$, if for every $m \in M, m \prec p$ then there exists some $q \in P$ such that $m \prec q \prec p$, for every $m \in M$. The pair $(P, \prec)$ is called an abstract basis, if $\prec$ is a transitive relation which also satisfies the interpolation property. A nonempty directed lower subset $I$ of $P$ is called a round ideal if for any $x \in I$ there is $y \in I$ such that $x \prec y$. The set of all round ideals of $P$ partially ordered by $\subseteq$ is called the ideal completion of $P$, denoted by $\operatorname{Idl}(P)$. Let $\uparrow p=\{q: p \prec q\}$ and $\downarrow p=\{q: q \prec p\}$. An auxiliary relation is called approximating if $\Downarrow p \subseteq \downarrow q$ implies $p \sqsubseteq q$. One can see that the set $\{\uparrow p: p \in P\}$ forms a basis for a topology called the pseudoScott topology on $P$, denoted by $\mathbf{P} \sigma$. The following fact is needed for the proof of Theorem 3.12 .

Fact 1.1. Let $(P, \sqsubseteq)$ be a poset with an auxiliary relation $\prec$. Then

(1) $(\operatorname{Idl}(P), \subseteq)$ is a continuous dcpo.

(2) If $\prec$ is approximating on $P$, then the map $j: P \rightarrow \operatorname{Idl}(P)$ defined by $j(p)=\star p$ is an embedding of $(P, \mathbf{P} \sigma)$ into $(\operatorname{Idl}(P), \sigma)$ where $\sigma$ denotes the Scott topology.

(3) If $\prec$ is approximating and all $\prec$-directed sets of $P$ have upper bounds, then $j(\max P)=$ $\max \operatorname{Idl}(P)$.

Proof. See KKW04, Theorem 2.3.

Below, we fix the key notion of a computational model for a $T_{0}$ topological space.

Before that, recall any $T_{0}$ topology $\tau$ on a space $X$ induces a partial order $\sqsubseteq_{\tau}$, called the specialization order, which is defined by

$$
x \sqsubseteq_{\tau} y \quad \Leftrightarrow \quad x \in c l_{\tau} y,
$$

for all $x, y \in X . c l_{\tau} y$ stands for the closure of $y$ with respect to $\tau$. Also, a partially ordered set $(P, \sqsubseteq)$ is an $\omega$-dcpo if every $\sqsubseteq$-ascending sequence has a least upper bound (see [Kni91]).

Definition 1.2. A triple $(P, \sqsubseteq, \phi)$ is a $(\omega-)$ computational model for $(X, \tau)$ whenever

(1) $(P, \sqsubseteq)$ is a continuous $(\omega-)$ dcpo.

(2) $\phi$ is a topological embedding from $(X, \tau)$ into $(P, \sqsubseteq)$ endowed with the Scott topology.

(3) $\phi\left(\operatorname{Max}\left(X, \sqsubseteq_{\tau}\right)\right)=\operatorname{Max}(P, \sqsubseteq)$.

Blanck in [Bla00] considered this definition as a domain representation for $(X, \tau)$ without mentioning the third condition. If we restrict ourselves to $T_{1}$ topological spaces, then the above definition coincides with the usual definition of computational model in which $\phi$ defines a homeomorphism from $(X, \tau)$ onto the space of maximal elements of $(P, \sqsubseteq)$ [KKW04, MMR02. 
Next, we define the notion of a quasi-metric space. For more details the reader may consult the references [FL82, Kel63, Kün95, Kü02, KS02]. A quasi-metric $d$ on a set $X$ is a function $d: X \times X \rightarrow[0, \infty)$ such that for any $x, y, z \in X$ :

(1) $x=y$ iff $d(x, y)=d(y, x)=0$,

(2) $d(x, z) \leq d(x, y)+d(y, z)$.

If we drop the if part of condition (1), $d$ is called a quasi-pseudometric. The pair $(X, d)$ is called quasi-(pseudo)metric space. Each quasi-metric $d$ on the set $X$ induces a $T_{0}$ topology on $X$, denoted by $\tau_{d}$, whose base is the set of all balls of the form $\mathcal{N}_{\epsilon}(x)=\{y \in X$ : $d(x, y)<\epsilon\}$, for any $x \in X$ and $\epsilon>0$. The topology $\tau_{d}$ is $T_{1}$ if and only if the condition (1) can be replaced by: $x=y \Leftrightarrow d(x, y)=0$. The quasi-metric $d$ generates another quasimetric $d^{-1}$ on the set $X$, called the conjugate of $d$, defined by $d^{-1}(x, y)=d(y, x)$. Also, the function $d^{*}$ can be defined on $X \times X$ by $d^{*}(x, y)=\max \left\{d(x, y), d^{-1}(x, y)\right\}$ which is a metric on $X$. The quasi-metric space $(X, d)$ is point symmetric if $\tau_{d} \subseteq \tau_{d^{-1}}$. For example, any compact $T_{1}$ quasi-metric space $(X, d)$ is point symmetric ([Wes57], Lemma 2). A sequence $\left(x_{n}\right)_{n>0}$ is called Cauchy (biCauchy) sequence if for every $\epsilon>0$ there is $N>0$ such that $d\left(x_{n}, x_{m}\right)<\epsilon$ whenever $m \geq n \geq N(m, n \geq N)$. An element $x \in X$ is called a Yoneda limit of the sequence $\left(x_{n}\right)_{n>0}$, if for any $y \in X$,

$$
d(x, y)=\inf _{n} \sup _{m \geq n} d\left(x_{m}, y\right) .
$$

The quasi-metric space $(X, d)$ is sequentially Yoneda-complete if every Cauchy sequence has a Yoneda limit. It is easy to see that the Yoneda limit is unique if it exists. A point $e \in X$ is called finite if for any Cauchy sequence $\left(x_{n}\right)_{n>0}$ in $X$ with the Yoneda limit $x$,

$$
d(e, x)=\sup _{n} \inf _{m \geq n} d\left(e, x_{m}\right) .
$$

The quasi-metric space $(X, d)$ is called algebraic if each element of $X$ is the Yoneda limit of a Cauchy sequence of finite elements. The quasi-metric space $(X, d)$ is Smyth-complete if any Cauchy sequence $\left(x_{n}\right)_{n>0}$ converges strongly in $X$, i.e. there is a point $x \in X$ such that $\left(x_{n}\right)_{n>0}$ converges to $x$ in the topology of the metric $d^{*}$.

Finally, we review some basic definitions from the hyperspace theory [CR06, RR02]. Let $(X, d)$ be a bounded quasi-metric space and $\mathcal{K}_{0}(X)$ denote the set of all nonempty compact subsets of $X$. The upper Hausdorff quasi-pseudometric $H_{d}^{+}$and the lower Hausdorff quasipseudometric $H_{d}^{-}$on $\mathcal{K}_{0}(X)$ are defined as follows:

$$
H_{d}^{+}(A, B)=\sup _{b \in B} d(A, b) \quad, \quad H_{d}^{-}(A, B)=\sup _{a \in A} d(a, B)
$$

for all $A, B \in \mathcal{K}_{0}(X)$, where $d(A, x)=\inf _{a \in A} d(a, x)$ and $d(x, A)=\inf _{a \in A} d(x, a)$. The Hausdorff quasi-pseudometric $H_{d}$ is defined as $H_{d}^{+} \vee H_{d}^{-}$or equivalently

$$
H_{d}(A, B)=\max \left\{\sup _{b \in B} d(A, b), \sup _{a \in A} d(a, B)\right\}
$$

for all $A, B \in \mathcal{K}_{0}(X)$. It is known that $H_{d}^{+}, H_{d}^{-}$and $H_{d}$ are quasi-pseudometrics on $\mathcal{K}_{0}(X)$. For a $T_{1}$ quasi-metric space $(X, d), H_{d}$ is a quasi-metric. Furthermore, for any $A, B \in \mathcal{K}_{0}(X)$,

$$
H_{d}(A, B)=0 \quad \text { if and only if } \quad B \subseteq A \subseteq c l_{\tau_{d}} B .
$$

In [AP10, the authors present an example which shows that $\left(\mathcal{K}_{0}(X), H_{d}\right)$ may not be a $T_{1}$ space, even though $(X, d)$ is a $T_{1}$ quasi-metric space. However, one can infer from $(*)$ 
that $\left(\mathcal{K}_{0}(X), H_{d}\right)$ is $T_{1}$ if $(X, d)$ is Hausdorff (more generally $\mathrm{KC}$-space in which all compact subsets are closed).

Recall that a subset $K$ of a quasi-metric space $(X, d)$ is $d$-precompact if for any $\epsilon>0$, there is a finite subset $F$ of $K$ such that for any $k \in K, d(x, k)<\epsilon$, for some $x \in F$. Unlike the metric spaces, a compact subset of a quasi-metric space $(X, d)$ is not necessarily $d^{-1}$ precompact. The following theorem shows that if we impose this extra condition to $(X, d)$, then the Smyth-completeness of $(X, d)$ can be lifted up to $\left(\mathcal{K}_{0}(X), H_{d}\right)$. This theorem is used in section 5, Lemma 4.14.

Theorem 1.3. Let $(X, d)$ be a Smyth-complete quasi-metric space such that any compact subset of $X$ is $d^{-1}$-precompact. Then $\left(\mathcal{K}_{0}(X), H_{d}\right)$ is Smyth-complete.

Proof. See [AP10], Theorem 3.7.

There are other topologies on the hyperspace $\mathcal{K}_{0}(X)$. The most famous of these topologies is the Vietoris topology $\tau_{V}$ which is the supremum of lower Vietoris topology and upper Vietoris topology. The lower Vietoris topology $\tau_{V}^{-}$is generated by all sets of the form $\diamond V=\left\{K \in \mathcal{K}_{0}(X): K \cap V \neq \emptyset\right\}$ whereas the upper Vietoris topology $\tau_{V}^{+}$is generated by all sets of the form $\square V=\left\{K \in \mathcal{K}_{0}(X): K \subseteq V\right\}$ for open $V$. In general, this topology is coarser than the topology of Hausdorff quasi-pseudometric $H_{d}$ on the hyperspace $\mathcal{K}_{0}(X)$. However, whenever any compact subset of $X$ is $d^{-1}$-precompact, these topologies coincide RR02].

\section{The SPACE OF FORMAL BALls AND ITS $\omega$-Plotkin DOMAIN}

The space of formal balls of a metric space $(X, d)$, denoted by $\mathbf{B} X$, was defined by Edalat and Heckmann in EH98. This construction gives a concrete computational model for metric spaces in which the order-theoretic properties of $\mathbf{B} X$ are closely connected with the metric properties of $(X, d)$.

In [Rut98, Rutten was probably the first who studied the space of formal balls for quasimetric spaces via co-Yoneda embedding. More recently, Ali-Akbari et al. in [AHPR09] and Romaguera and Valero in [RV09, RV10] studied the set of formal balls for quasi-metric spaces in the spirit of Edalat and Heckmann's work.

Definition 2.1. For a quasi-metric space $(X, d)$, the space of formal balls is the pair $(\mathbf{B} X, \sqsubseteq)$ where

$$
\mathbf{B} X=\{(x, r): x \in X \text { and } r \geq 0\},
$$

and

$$
(x, r) \sqsubseteq(y, s) \quad \text { if and only if } \quad d(x, y) \leq r-s,
$$

for any $(x, r),(y, s) \in \mathbf{B} X$. It is easy to see that $(\mathbf{B} X, \sqsubseteq)$ is a poset. An element $(x, r)$ of $\mathbf{B} X$ is called a formal ball. One can define an auxiliary relation $\prec$ on $\mathbf{B} X$ as follows:

$$
(x, r) \prec(y, s) \quad \text { if and only if } \quad d(x, y)<r-s .
$$

It can be shown that the relation $\prec$ satisfies the interpolation property and therefore $(\mathbf{B} X, \prec)$ forms an abstract basis.

The following theorem shows some interesting properties of the poset of formal balls.

Theorem 2.2. Let $(X, d)$ be a quasi-metric space.

(1) The function $\iota:\left(X, \tau_{d}\right) \rightarrow(\mathbf{B} X, \mathbf{P} \sigma)$ defined by $\iota(x)=(x, 0)$ is an embedding. 
(2) If $(X, d)$ is $T_{1}$ and sequentially Yoneda-complete, then $(\mathbf{B} X, \sqsubseteq)$ is a dcpo. In addition, if $(X, d)$ is also algebraic, $(\mathbf{B} X, \sqsubseteq)$ is a domain (continuous dcpo).

(3) If $(X, d)$ is Smyth-complete, then $(\mathbf{B} X, \sqsubseteq)$ is a domain. Moreover, the auxiliary relation $\prec$ coincides with the way-below relation of $\mathbf{B} X$.

Proof. See Theorem 3.12, Corollary 3.13 and Theorem 3.17 in [AHPR09.

In the light of the first part of the above Theorem, to ease our notations, we identify the set $X$ with the set $\iota(X)=\{(x, 0): x \in X\}$. In particular, any compact subset of $X$ is identified with a compact subset of the set $\iota(X)$.

Now, we review the definition of chain completion of an abstract basis $(P, \prec)$. The set of all $\omega$-chains, i.e. $\prec$-ascending sequences, of $P$ is denoted by $C P$.

Definition 2.3. For two $\omega$-chains $\left(x_{n}\right)_{n>0}$ and $\left(y_{m}\right)_{m>0}$ in $C P$ define

$$
\begin{gathered}
\left(x_{n}\right)_{n>0} \sqsubseteq\left(y_{m}\right)_{m>0} \Leftrightarrow \forall n \exists m \quad x_{n} \prec y_{m}, \\
\left(x_{n}\right)_{n>0} \sim\left(y_{m}\right)_{m>0} \Leftrightarrow\left(x_{n}\right)_{n>0} \sqsubseteq\left(y_{m}\right)_{m>0} \&\left(y_{m}\right)_{m>0} \sqsubseteq\left(x_{n}\right)_{n>0} .
\end{gathered}
$$

The chain completion of the abstract basis $(P, \prec)$ is defined to be the partially ordered set $(\mathcal{C} P, \sqsubseteq)$ where $\mathcal{C} P=C P / \sim$ and

$$
\left[\left(x_{n}\right)_{n>0}\right] \sqsubseteq\left[\left(y_{m}\right)_{m>0}\right] \Leftrightarrow\left(x_{n}\right)_{n>0} \sqsubseteq\left(y_{m}\right)_{m>0},
$$

for any $\left[\left(x_{n}\right)_{n>0}\right]$ and $\left[\left(y_{m}\right)_{m>0}\right]$ in $\mathcal{C} P$. It is a well-known fact that $(\mathcal{C} P, \sqsubseteq)$ is a continuous $\omega$-dcpo [Kni91]. The way-below relation is given by:

$$
\left[\left(x_{n}\right)_{n>0}\right] \ll\left[\left(y_{m}\right)_{m>0}\right] \Leftrightarrow \exists m \forall n \quad x_{n} \prec y_{m} .
$$

$\left(x_{n}\right)_{n>0}$ is called a representation of $\left[\left(x_{n}\right)_{n>0}\right] \in \mathcal{C} P$. By abuse of notation, for any equivalence class $\mathcal{I}$ of $\mathcal{C} P$, we write $x \in \mathcal{I}$ if $x$ is an element of one of the sequences representing $\mathcal{I}$.

Definition 2.4. For subsets $A$ and $B$ of the abstract basis $(P, \prec)$, define

$$
\begin{aligned}
& A \prec_{U} B \Leftrightarrow \forall b \in B \exists a \in A \quad a \prec b, \\
& A \prec_{L} B \Leftrightarrow \forall a \in A \exists b \in B \quad a \prec b, \\
& A \prec_{E M} B \text { if and only if } A \prec_{U} B \text { and } A \prec_{L} B .
\end{aligned}
$$

The relation $\prec_{E M}$ stands for Egli-Milner relation. Let $\mathcal{P}_{\text {fin }} P$ be the set of all non-empty finite subsets of $P$. It is easy to see that $\left(\mathcal{P}_{f i n} P, \prec_{E M}\right)$ is an abstract basis. Since there is no danger of confusion, for brevity, we drop the subscript EM. The chain completion of $\left(\mathcal{P}_{\text {fin }} P, \prec\right)$ is called $\omega$-Plotkin domain of $P$ which is denoted by $\mathbf{C} P$.

In particular, for a quasi-metric space $(X, d)$, we consider the $\omega$-Plotkin domain $\mathbf{C B} X$ of the abstract basis $(\mathbf{B} X, \prec)$. For $F \in \mathcal{P}_{\text {fin }} \mathbf{B} X$ and $\mathcal{I} \in \mathbf{C B} X$, define

$$
r F=\max \{r:(x, r) \in F\} \text { and } \bar{r} \mathcal{I}=\inf \{r F: F \text { belongs to a representation of } \mathcal{I}\} .
$$

Note that $\mathcal{I} \sqsubseteq \mathcal{J}$ implies that $\bar{r} \mathcal{I} \geq \bar{r} \mathcal{J}$. Also from $F \prec G, r F>r G$ follows. Therefore, if $\mathcal{I} \ll \mathcal{J}$ then $\bar{r} \mathcal{I}>\bar{r} \mathcal{J}$.

Below, an important property of this structure is highlighted.

Theorem 2.5. Let $(X, d)$ be a quasi-metric space. Then $\omega$-Plotkin domain $(\mathbf{C B} X, \sqsubseteq)$ is a continuous $\omega$-dcpo and moreover, any $\ll$-directed subset of it has a least upper bound. 
Proof. As we mentioned earlier, the first part is known. For the second part, let $\preceq$ be the partial order relation generated by $\ll$, i.e.

$$
\mathcal{I} \preceq \mathcal{J} \quad \text { if and only if } \quad \mathcal{I}=\mathcal{J} \text { or } \mathcal{I} \ll \mathcal{J},
$$

for every $\mathcal{I}, \mathcal{J} \in \mathbf{C B} X$.

We first verify that $(\mathbf{C B} X, \preceq)$ is a dcpo. By a well-known fact from Mark78] it suffices to examine that every $\preceq$-chain of $\mathbf{C B} X$ has a least upper bound. Let $\mathcal{A}=\left(\mathcal{I}_{\alpha}\right)_{\alpha \in I}$ be a $\preceq$-chain in $\mathbf{C B} X$. Without loss of generality, we may assume that $\mathcal{A}$ has no maximum element. Then $\left(\bar{r} \mathcal{I}_{\alpha}\right)_{\alpha \in I}$ is a strictly decreasing chain in the set of nonnegative real numbers and therefore has an infimum, say $r$. Fix $\mathcal{I}_{1}$ and inductively for any $n \geq 2$, choose $\mathcal{I}_{n-1} \ll \mathcal{I}_{n}$ such that $r<\bar{r} \mathcal{I}_{n}<r+\frac{1}{n}$. We claim that $\left(\mathcal{I}_{n}\right)_{n>0}$ is a cofinal subsequence of $\mathcal{A}$ in $\mathbf{C B} X$. Let $\mathcal{I}_{\alpha}$ be an arbitrary element of $\mathcal{A}$. Then choose $\mathcal{I}_{n}$ in such a way that $\bar{r} \mathcal{I}_{n}<\bar{r} \mathcal{I}_{\alpha}$. Now since any two elements of $\mathcal{A}$ are comparable and $\bar{r} \mathcal{I}_{n}<\bar{r} \mathcal{I}_{\alpha}$, it follows that $\mathcal{I}_{\alpha} \ll \mathcal{I}_{n}$. But $(\mathbf{C B} X, \sqsubseteq)$ is an $\omega$-dcpo. Therefore, $\left(\mathcal{I}_{n}\right)_{n>0}$ has the least upper bound $\mathcal{I}$ in $(\mathbf{C B} X, \sqsubseteq)$, which is also the least upper bound for $\mathcal{A}$.

Now, let $\mathcal{D}$ be a $\ll$-directed subset of $\mathbf{C B} X$. Then it is easy to see that $\mathcal{D}$ is also $\preceq$-directed. Therefore, it has the $\preceq$-least upper bound $\mathcal{I}$. It is, then, straightforward to show that $\mathcal{I}$ is also the «-least upper bound of $\mathcal{D}$.

Although we are not able to show that $\mathbf{C B} X$ is a dcpo, the above Theorem gives a crucial feature of $\mathbf{C B} X$ which will help us in obtaining Theorem 3.12 .

We introduce the following abbreviations which will be useful for a number of later proofs.

Note 2.6. Let $F, G \in \mathcal{P}_{\text {fin }} \mathbf{B} X$ and $\epsilon>0$ be given.

(1) $F+\epsilon=\{(x, r+\epsilon):(x, r) \in F\}$.

(2) If $F \prec G$, then put $\delta(F, G)=\min \{(r-s)-d(x, y):(x, r) \in F,(y, s) \in G,(x, r) \prec$ $(y, s)\}$.

Remark 2.7. The following properties of the above notations are straightforward.

(1) For $0<\epsilon^{\prime}<\epsilon, F+\epsilon \prec F+\epsilon^{\prime} \prec F$.

(2) For any $\epsilon<\delta(F, G),(x, r) \in F$ and $(y, s) \in G$, with $(x, r) \prec(y, s)$, we have $(x, r) \prec$ $(y, s+\epsilon)$. Hence $F \prec G+\epsilon$.

\section{Embedding of $\mathcal{K}_{0}(X)$ into $\mathbf{C B} X$}

In this section, we apply the techniques used by Edalat and Heckmann in [EH98] to the $\omega$-Plotkin domain of the space of formal balls, leading us to find a computational model for the space $\mathcal{K}_{0}(X)$ of the nonempty compact subsets of a quasi-metric space $(X, d)$. More precisely, for a sequentially Yoneda-complete $T_{1}$ quasi-metric space $(X, d)$, we construct the $\omega$-Plotkin domain of the abstract basis $(\mathbf{B} X, \prec)$, as introduced in Definition 2.1, and show that the hyperspace $\mathcal{K}_{0}(X)$ equipped with the Vietoris topology $\tau_{V}$ can be embedded in $\mathbf{C B} X$ equipped with the Scott topology. Moreover, this embedding serves as an $\omega$ computational model of $\left(\mathcal{K}_{0}(X), \tau_{V}\right)$.

From now on, we assume that $(X, d)$ is a sequentially Yoneda-complete $T_{1}$ bounded quasi-metric space. 
Definition 3.1. Let $K$ be a nonempty compact subset of $X$. Since $K$ is compact, it is $d$-precompact. So, for any $n>0$, one can choose $x_{1}^{n}, \ldots, x_{m_{n}}^{n}$ of $K$ such that for any $x \in K$, $d\left(x_{i}^{n}, x\right)<\frac{1}{2 n^{2}}$ for some $x_{i}^{n}$. Put

$$
F_{n}=\left\{\left(x_{1}^{1}, \frac{1}{n}\right), \ldots,\left(x_{m_{1}}^{1}, \frac{1}{n}\right), \ldots,\left(x_{1}^{n}, \frac{1}{n}\right), \ldots,\left(x_{m_{n}}^{n}, \frac{1}{n}\right)\right\} .
$$

It can be easily checked that $\left(F_{n}\right)_{n>0}$ is an $\omega$-chain in $\left(\mathcal{P}_{f i n}(\mathbf{B} X), \prec\right)$. Call $\left(F_{n}\right)_{n>0}$ a standard representation of $K$.

Although a compact subset $K$ of $X$ might have several standard representations, we show that all standard representations of $K$ are $\sim$-equivalent. The following auxiliary lemmas will be useful in several proofs.

Lemma 3.2. For any $x \in K$ and any standard representation $\left(F_{n}\right)_{n>0}$ of $K$, there is a sequence $\left(\left(x_{n}, r_{n}\right)\right)_{n>0}$ such that $\left(x_{n}, r_{n}\right) \in F_{n}$ and $d^{*}\left(x_{n}, x\right) \rightarrow 0$.

Proof. First note that for any $n>0, F_{n} \prec K$. Hence for any $n>0$, there is $\left(x_{n}, r_{n}\right) \in F_{n}$ such that $\left(x_{n}, r_{n}\right) \prec(x, 0)$. As $r F_{n} \rightarrow 0$, it implies $d\left(x_{n}, x\right) \rightarrow 0$. Now, since $K$ is compact and therefore $d$ is point symmetric on $K$, it follows $d\left(x, x_{n}\right) \rightarrow 0$. Therefore $d^{*}\left(x_{n}, x\right) \rightarrow 0$.

Let $\mathcal{I}$ be an element of $\mathbf{C B} X$. We can obtain a nonempty compact saturated subset of $\mathbf{B} X$, denoted by $\mathcal{I}^{+}$, as

$$
\mathcal{I}^{+}=\bigcap_{n>0} \uparrow F_{n}
$$

where $\left(F_{n}\right)_{n>0}$ is a representation of $\mathcal{I}$. In fact, since $\mathcal{I}^{+}$is a filtered intersection of nonempty compact saturated subsets of $(\mathbf{B} X, \mathbf{P} \sigma)$ and $\mathbf{B} X$ is a dcpo, similar to Theorem 7.2.27 in AJ94, it can be proved that the pseudoScott topology $\mathbf{P} \sigma$ is sober. So $\mathcal{I}^{+}$is a nonempty compact saturated subset of $\mathbf{B} X$. Also, $\mathcal{I}^{+}$is independent of the choice of its representations. One can easily show that for any representations $\left(F_{n}\right)_{n>0}$ and $\left(E_{m}\right)_{m>0}$ of $\mathcal{I}$,

$$
\bigcap_{n} \uparrow F_{n}=\bigcap_{m} \uparrow E_{m} .
$$

Lemma 3.3. Let $\mathcal{I}$ be in $\mathbf{C B} X$ with $\bar{r} \mathcal{I}=0$. Then for any standard representation $\left(F_{n}\right)_{n>0}$ of $\iota^{-1}\left(\mathcal{I}^{+}\right)$and any $G, H \in \mathcal{P}_{\text {fin }} \mathbf{B} X$ with $G \prec H \prec \mathcal{I}^{+}, G \sqsubseteq F_{n}$ for some $n>0$.

Proof. Let $(y, s) \in G$. Since $G \prec \mathcal{I}^{+}$, there is $(x, 0) \in \mathcal{I}^{+}$such that $(y, s) \prec(x, 0)$. Now as $(x, 0) \in \bigcap_{n>0} \uparrow F_{n}$, by Lemma 3.2. there is a sequence $\left(\left(x_{n}, r_{n}\right)\right)_{n>0}$ such that $\left(x_{n}, r_{n}\right) \in F_{n}$ and $d^{*}\left(x_{n}, x\right) \rightarrow 0$. For $\epsilon=s-d(y, x)$, take $n>0$ such that $d\left(x, x_{n}\right)<\epsilon / 2$ and $r_{n}<\epsilon / 2$. One can readily see that $(y, s) \prec\left(x_{n}, r_{n}\right)$. So, for any $(y, s) \in G$, there is $F_{n}$ and $\left(x_{n}, r_{n}\right) \in F_{n}$ such that $(y, s) \prec\left(x_{n}, r_{n}\right)$. Assume that $F$ in $\left(F_{n}\right)_{n>0}$ is an upper bound for all $F_{n}$, arisen in this way. Now, $G \sqsubseteq_{L} F$ is straightforward.

Next, put $\delta=\delta(G, H)$, as defined in Notation [2.6. Choose $F_{n}$ such that $r F_{n}<\delta$ and $F \prec F_{n}$. Take $(x, r) \in F_{n}$. Then $(x, 0) \in \mathcal{I}^{+}$. Therefore, there exists $(z, t) \in H$ such that $(z, t) \prec(x, 0)$. Also, $G \prec_{U} H$ implies that there exists $(y, s) \in G$ such that $(y, s) \prec(z, t)$. It follows that

$$
\begin{aligned}
d(y, x) & \leq d(y, z)+d(z, x) \\
& \leq(s-t)-\delta+t \\
& <s-r .
\end{aligned}
$$

Hence $G \prec_{U} F_{n}$. Now from $G \sqsubseteq_{L} F$ and $F \prec F_{n}$, it follows that $G \sqsubseteq_{L} F_{n}$ and consequently $G \sqsubseteq F_{n}$. 
Proposition 3.4. For any $K \in \mathcal{K}_{0}(X)$, all standard representations of $K$ are $\sim$-equivalent.

Proof. Let $\left(F_{n}\right)_{n>0}$ and $\left(E_{m}\right)_{m>0}$ be two standard representations of $K$. For any $m>0$, since $E_{m} \prec E_{m+1} \prec K$, it follows from Lemma 3.3 that there is $n>0$ such that $E_{m} \prec F_{n}$. Therefore $\left(E_{m}\right)_{m>0} \sqsubseteq\left(F_{n}\right)_{n>0}$. Similarly, it can be proved that $\left(F_{n}\right)_{n>0} \sqsubseteq\left(E_{m}\right)_{m>0}$ and therefore $\left(E_{m}\right)_{m>0}$ and $\left(F_{n}\right)_{n>0}$ are in an equivalence class.

In the light of the above proposition, the following definition is established.

Definition 3.5. Denote the equivalence class of a standard representation $\left(F_{n}\right)_{n>0}$ of a nonempty compact subset $K$ by $K^{*}$ and let $\phi: \mathcal{K}_{0}(X) \rightarrow \mathbf{C B} X$ be $\phi(K)=K^{*}$.

We prove some properties of this map.

Proposition 3.6. Let $K, L \in \mathcal{K}_{0}(X)$ and $\mathcal{I} \in \mathbf{C B} X$ with $\bar{r} \mathcal{I}=0$. Then

(1) $K=\left(K^{*}\right)^{+}$.

(2) For any representation $\left(F_{n}\right)_{n>0}$ of $\mathcal{I}$;

$$
\mathcal{I}^{+}=\left\{\bigsqcup_{n} a_{n}: a_{n} \in F_{n},\left(a_{n}\right)_{n>0} \text { is an ascending sequence }\right\} .
$$

(3) $K^{*} \sqsubseteq L^{*}$ implies $L \subseteq K \subseteq \operatorname{cl}_{\tau_{d}} L$, where $c l_{\tau_{d}} L$ is the closure of $L$ in $\tau_{d}$.

Proof. (1) It is routine to check that $K \subseteq\left(K^{*}\right)^{+}$. For the opposite inclusion, suppose that there is $(x, 0) \in\left(K^{*}\right)^{+} \backslash K$. For any $(y, 0) \in K$, put $s_{y}=\frac{1}{2} d(y, x)$. Using compactness of $K$, choose a finite subset $G_{0}$ of $\left\{\left(y, \frac{1}{2} s_{y}\right):(y, 0) \in K\right\}$ such that $G_{0} \prec K$. Select $F \in K^{*}$ with $r F<\min \left\{\frac{1}{2} s_{y}:\left(y, \frac{1}{2} s_{y}\right) \in G_{0}\right\}$. We claim that $G \prec_{U} F$, where $G=\left\{\left(y, s_{y}\right)\right.$ : $\left.\left(y, \frac{1}{2} s_{y}\right) \in G_{0}\right\}$. To prove the claim, let $(z, t) \in F$. So $(z, 0) \in K$ and therefore there is $\left(y, \frac{1}{2} s_{y}\right) \in G_{0}$ such that $\left(y, \frac{1}{2} s_{y}\right) \prec(z, 0)$. Since $t<\frac{1}{2} s_{y}$, it follows $\left(y, s_{y}\right) \prec(z, t)$ and consequently $G \prec_{U} F$.

Now, by definition of $\left(K^{*}\right)^{+}$, it is clear that $F \prec_{U}\left(K^{*}\right)^{+}$. So there is $(a, u) \in F$ with $(a, u) \prec(x, 0)$. Since $G \prec_{U} F$, there is $\left(y, s_{y}\right)$ in $G$ such that $\left(y, s_{y}\right) \prec(a, u)$. Hence $\left(y, s_{y}\right) \prec(x, 0)$ or equivalently $d(y, x)<s_{y}$, which is a contradiction.

(2) Clearly the supremum of any ascending sequence $\left(a_{n}\right)_{n>0}$, where $a_{n} \in F_{n}$, belongs to $\mathcal{I}^{+}$. Let $(a, 0) \in \mathcal{I}^{+}$. Put $G_{n}=\left\{(x, r) \in F_{n}:(x, r) \prec(a, 0)\right\}$. For any $(x, r)$ and $(y, s)$ in $\bigcup_{n} G_{n}$, define $(x, r) R(y, s)$ if and only if for some $n>0,(x, r) \in G_{n},(y, s) \in G_{n+1}$ and $(x, r) \prec(y, s)$. The binary relation $R$ defines a locally finite directed graph on the infinite set $G=\bigcup_{n} G_{n}$ with at most $\left|F_{1}\right|$-connected components. So $G$ has an infinite connected component and therefore, in the light of König's Lemma, there is an ascending sequence $\left(\left(x_{n}, r_{n}\right)\right)_{n>0}$ such that $\bigsqcup_{n}\left(x_{n}, r_{n}\right)=(b, 0) \sqsubseteq(a, 0)$. Since $d$ is $T_{1}, b=a$ follows and the proof is complete.

(3) The assumption implies $\left(L^{*}\right)^{+} \subseteq\left(K^{*}\right)^{+}$. So in the light of the first part, $L \subseteq K$. For $K \subseteq c l_{\tau_{d}} L$, let $x \in K$. Suppose that $\left(F_{n}\right)_{n>0}$ and $\left(G_{n}\right)_{n>0}$ are standard representations of $K$ and $L$, respectively. By the second part, there is an ascending sequence $\left(\left(x_{n}, r_{n}\right)\right)_{n>0}$, $\left(x_{n}, r_{n}\right) \in F_{n}$, such that $\bigsqcup_{n}\left(x_{n}, r_{n}\right)=(x, 0)$. So $d\left(x_{n}, x\right) \rightarrow 0$. Since $K$ is compact and therefore $d$ is point symmetric on $K, d\left(x, x_{n}\right) \rightarrow 0$. From $K^{*} \sqsubseteq L^{*}$, it follows that for any $F_{n}$, there is $G_{m_{n}}$ and $\left(y_{m_{n}}, s_{m_{n}}\right) \in G_{m_{n}}$ such that $F_{n} \sqsubseteq G_{m_{n}}$ and $\left(x_{n}, r_{n}\right) \prec\left(y_{m_{n}}, s_{m_{n}}\right)$. Thus $d\left(x, y_{m_{n}}\right) \rightarrow 0$, which means $x \in c l_{\tau_{d}} L$. 
From the first part of the above proposition, it follows that the map $\phi: \mathcal{K}_{0}(X) \rightarrow \mathbf{C B} X$ is one-to-one. Moreover, in the following we prove that the map $\phi$ gives a one-to-one correspondence between the maximal element of $\mathcal{K}_{0}(X)$ with respect to the specialization order $\sqsubseteq_{d}$ and the maximal element of the partially ordered set $\mathbf{C B} X$. Before proving this, we need the following lemma.

Lemma 3.7. For any maximal element $\mathcal{I}$ of $\mathbf{C B} X, \mathcal{I}=I^{*}$ where $I=\iota^{-1}\left(\mathcal{I}^{+}\right)$.

Proof. Note that $\mathcal{I}^{+}$is a nonempty compact subset of $\mathbf{B} X$. Since $\iota:\left(X, \tau_{d}\right) \rightarrow(\mathbf{B} X, \mathbf{P} \sigma)$ is an embedding (Theorem 2.2),$I=\iota^{-1}\left(\mathcal{I}^{+}\right)$is a compact set in $\left(X, \tau_{d}\right)$. Thus $I^{*}$ is well-defined.

Now, by maximality of $\mathcal{I}$, it suffices to prove that $\mathcal{I} \sqsubseteq I^{*}$. Let $\left(F_{n}\right)_{n>0}$ be a representations of $\mathcal{I}$ and $\left(G_{m}\right)_{m>0}$ be a standard representation of $I$. Take $F_{n}$ in $\left(F_{n}\right)_{n>0}$. Since $F_{n} \prec F_{n+1} \prec \mathcal{I}^{+}$, according to Lemma $3.3, F_{n} \sqsubseteq G_{m}$ for some $m>0$. This shows $\mathcal{I} \sqsubseteq I^{*}$.

Proposition 3.8. For any maximal element $K$ of $\mathcal{K}_{0}(X)$ with respect to the specialization order $\sqsubseteq_{H_{d}}, K^{*}$ is maximal in $(\mathbf{C B} X, \sqsubseteq)$. Conversely, any maximal element $\mathcal{I}$ of $\mathbf{C B} X$ is of the form $K^{*}$ for some maximal element $K$ of $\left(\mathcal{K}_{0}(X), \sqsubseteq_{H_{d}}\right)$.

Proof. Let $K$ be a maximal element of $\left(\mathcal{K}_{0}(X), \sqsubseteq H_{d}\right)$ and $K^{*} \sqsubseteq \mathcal{I}$. Without loss of generality, we assume that $\mathcal{I}$ is maximal. So $\mathcal{I}=I^{*}$, where $I=\iota^{-1}\left(\mathcal{I}^{+}\right)$. We have $K^{*} \sqsubseteq I^{*}$ and therefore by the third part of Proposition 3.6, $I \subseteq K \subseteq c l_{\tau_{d}} I$. Hence $H_{d}(K, I)=0$. By maximality of $K$, we conclude that $K=I$ and $K^{*}=\mathcal{I}$.

Now, let $\mathcal{I}$ be a maximal element of $\mathbf{C B} X$. Lemma 3.7 implies that for any maximal element $\mathcal{I}$ of $\mathbf{C B} X, \mathcal{I}=I^{*}$, where $I=\iota^{-1}\left(\mathcal{I}^{+}\right)$is a nonempty compact subset of $(X, d)$. To complete the proof, we have to show that $I$ is maximal in $\left(\mathcal{K}_{0}(X)\right.$, $\left.H_{d}\right)$. Let $I \sqsubseteq H_{d} J$ for some $J \in \mathcal{K}_{0}(X)$. Thus $H_{d}(I, J)=0$ and consequently $J \subseteq I \subseteq c l_{\tau_{d}} J$. We show that $I^{*} \sqsubseteq J^{*}$. Suppose $\left(F_{n}\right)_{n>0}$ and $\left(G_{m}\right)_{m>0}$ are standard representations of $I$ and $J$, respectively. Since $J \subseteq I \subseteq c l_{\tau_{d}} J$, it follows that $F_{n} \prec F_{n+1} \prec J$, for any $n>0$. By Lemma 3.3. $F_{n} \sqsubseteq G_{m}$ for some $m>0$. So $I^{*} \sqsubseteq J^{*}$ and by maximality of $I^{*}$ it follows that $I=J$. Therefore $I$ is maximal in $\left(\mathcal{K}_{0}(X), \sqsubseteq H_{d}\right)$.

Below we examine different topologies on $\mathcal{K}_{0}(X)$ for which the map $\phi$ becomes an embedding.

Theorem 3.9. The map $\phi$ is an embedding from the hyperspace $\mathcal{K}_{0}(X)$ equipped with the Vietoris topology into $\mathbf{C B} X$ with the Scott topology.

Proof. Let $\Uparrow \mathcal{I}=\{\mathcal{J} \in \mathbf{C B} X: \mathcal{I} \ll \mathcal{J}\}$ be a basic open set of $\mathbf{C B} X$ in the Scott topology and $K \in \phi^{-1}(\Uparrow \mathcal{I})$. So $K^{*} \in \Uparrow \mathcal{I}$ or equivalently $\mathcal{I} \ll K^{*}$. Let $\left(F_{n}\right)_{n>0}$ be a standard representation of $K^{*}$. By definition of the way-below relation, there is $N>0$ such that for any element $G \in \mathcal{I}, G \prec F_{N-1}$. Define an open subset $\mathcal{V}$ of $\left(\mathcal{K}_{0}(X), \tau_{V}\right)$ as $\mathcal{V}=\left(\bigcap_{(x, r) \in F_{N}} \diamond V_{x}\right) \cap \square V$, where $V_{x}=\mathcal{N}_{\frac{1}{N}}(x)$ and $V=\bigcup_{(x, r) \in F_{N}} \mathcal{N}_{\frac{1}{N}}(x)$. Clearly $K \in \mathcal{V}$. We show that $\mathcal{V} \subseteq \phi^{-1}(\Uparrow \mathcal{I})$. Let $B \in \mathcal{V}$. First, we prove that $F_{N} \prec B$. Take $\left(x, \frac{1}{N}\right) \in F_{N}$. From $B \in \diamond V_{x}$, it follows that there is $b \in B \cap V_{x}$. Thus $d(x, b)<\frac{1}{N}$ or equivalently $\left(x, \frac{1}{N}\right) \prec(b, 0)$ and therefore $F_{N} \prec_{L} B$. Finally $F_{N} \prec_{U} B$ follows from $B \in \square V$. Now, by Lemma 3.3. for any standard representation of $B$, there is an element $H$ in this representation such that $F_{N-1} \prec H$. So for any element $G \in \mathcal{I}, G \prec H$. That means $\mathcal{I} \ll B^{*}$.

On the other hand, we prove that the image of any upper (resp. lower) Vietoris open subset under the map $\phi$ is open in the relative Scott topology on $\phi\left(\mathcal{K}_{0}(X)\right)$. Let $\square V$ 
be an upper Vietoris open subset of $\mathcal{K}_{0}(X)$ and $K^{*} \in \phi(\square V)$. Take $\epsilon>0$ such that $\bigcup_{x \in K} \mathcal{N}_{\epsilon}(x) \subseteq V$. Assume that $\left(F_{n}\right)_{n>0}$ is a standard representation of $K$. Choose $N>0$ such that $r F_{N}<\epsilon$ and put $\mathcal{I}_{K}=\left[\left(E_{n}\right)_{n>0}\right]$, where $E_{n}=F_{N}+\frac{1}{n}$ (See Notation [2.6.). Remark 2.7(1), indicates that the sequence $\left(E_{n}\right)_{n>0}$ is $\prec$-ascending and therefore $\left(E_{n}\right)_{n>0}$ is an $\omega$-chain. Clearly $\mathcal{I}_{K} \ll K^{*}$. We prove

$$
\Uparrow \mathcal{I}_{K} \cap \phi\left(\mathcal{K}_{0}(X)\right) \subseteq \phi(\square V) .
$$

Let $B^{*} \in \Uparrow \mathcal{I}_{K}$. We show that $B^{*} \in \phi(\square V)$ or equivalently $B \subseteq V$. Let $b \in B$. Because of $\mathcal{I}_{K} \ll B^{*}$, there is an element $H \in B^{*}$ such that $E_{n} \prec H$, for any $n>0$. So there are $(z, t) \in H$ and $\left(x_{n}, r_{n}+1 / n\right) \in E_{n}$, for any $n>0$, with $\left(x_{n}, r_{n}+1 / n\right) \prec(z, t) \prec(b, 0)$. Since the set $\left\{\left(x_{n}, r_{n}\right):\left(x_{n}, r_{n}+1 / n\right) \prec(b, 0)\right\} \subseteq F_{N}$ is finite, it follows that there is $(x, r) \in F_{N}$ such that $d(x, b) \leq r<\epsilon$. Thus from $x \in K, b \in V$ follows and consequently $B^{*} \in \phi(\square V)$.

Now, let $\diamond V$ be a lower Vietoris open subset of $\mathcal{K}_{0}(X)$. Suppose $K^{*} \in \phi(\diamond V)$ and $\left(F_{n}\right)_{n>0}$ is a standard representation of $K^{*}$. Select $x \in K \cap V$ and put $F_{n}^{\prime}=F_{n} \cup\{(x, 1 / n)\}$. One can readily see that $\left(F_{n}^{\prime}\right)_{n>0}$ is equivalent to $\left(F_{n}\right)_{n>0}$ and therefore belongs to $K^{*}$. Choose $N>0$ such that $\mathcal{N}_{\frac{1}{N}}(x) \subseteq V$. Define $\mathcal{I}_{K}=\left[\left(E_{n}\right)_{n>0}\right]$, where $E_{n}=F_{N}^{\prime}+\frac{1}{n}$. For any $n>0, E_{n} \prec F_{N}^{\prime}$. Thus $K^{*} \in \Uparrow \mathcal{I}_{K}$. We prove

$$
\Uparrow \mathcal{I}_{K} \cap \phi\left(\mathcal{K}_{0}(X)\right) \subseteq \phi(\diamond V) .
$$

Assume that $\mathcal{I}_{K} \ll B^{*}$. There is $H \in B^{*}$ such that for any $n>0, E_{n} \prec H \prec B$. Hence, one can find $(b, 0) \in B$ with $\left(x, \frac{1}{N}\right) \prec(b, 0)$. Thus $b \in \mathcal{N}_{\frac{1}{N}}(x)$ and $b \in V$. That implies $B^{*} \in \phi(\diamond V)$.

It is known that for any quasi-metric space $(X, d)$ whose compact subsets are $d^{-1}$ precompact, the Vietoris topology on $\mathcal{K}_{0}(X)$ coincides with the topology of the Hausdorff quasi-pseudometric $H_{d}$ ([RR02], Theorem 5). So in the light of Theorem 3.9, under this assumption, the map $\phi:\left(\mathcal{K}_{0}(X), H_{d}\right) \rightarrow(\mathbf{C B} X, \sigma)$ is an embedding. In the following, we present an alternative proof which avoids this well-known result.

Theorem 3.10. Let $(X, d)$ be a sequentially Yoneda-complete $T_{1}$ quasi-metric space such that any compact subset of $(X, d)$ is $d^{-1}$-precompact. Then the map $\phi:\left(\mathcal{K}_{0}(X), H_{d}\right) \rightarrow$ $(\mathbf{C B} X, \sigma)$ is an embedding.

Proof. Let $\Uparrow \mathcal{I}$ be a basic open set of $\mathbf{C B} X$ in the Scott topology. Let $K_{0}^{*} \in \Uparrow \mathcal{I}$ and $\left(F_{n}\right)_{n>0}$ be a representation of $K_{0}^{*}$. By definition of the way-below relation, there is a natural number $N>0$ such that for any $G \in \mathcal{I}, G \prec F_{N}$. Put $\epsilon=\frac{1}{2} \delta\left(F_{N}, F_{N+1}\right)$. To complete the proof of continuity of $\phi$, it suffices to show that

$$
\mathcal{N}_{\epsilon}\left(K_{0}\right) \subseteq \phi^{-1}(\Uparrow \mathcal{I}) .
$$

Let $K \in \mathcal{N}_{\epsilon}\left(K_{0}\right)$. Define $F^{\prime}=F_{N+1}+\epsilon$. By Remark 2.7 (2), $F_{N} \prec F^{\prime}$.

We prove that $F^{\prime} \prec K$. For this, take $(y, s+\epsilon) \in F^{\prime}$. Hence $(y, s) \in F_{N+1}$ and by $F_{N+1} \prec K_{0}$, there is an element $x \in K_{0}$ such that $(y, s) \prec(x, 0)$. Since $H_{d}\left(K_{0}, K\right)<\epsilon$, there is an $x^{*} \in K$ such that $d\left(x, x^{*}\right)<\epsilon$. Thus

$$
d\left(y, x^{*}\right) \leq d(y, x)+d\left(x, x^{*}\right)<s+\epsilon .
$$

Consequently $(y, s+\epsilon) \prec\left(x^{*}, 0\right)$ and $F^{\prime} \prec_{L} K$. A similar argument shows that $F^{\prime} \prec_{U} K$ and therefore $F^{\prime} \prec K$ is established. Now, by Lemma 3.3, since $F_{N} \prec F^{\prime} \prec K$, there is $H \in K^{*}$ such that $F_{N} \sqsubseteq H$. Thus for any $G \in \mathcal{I}, G \prec H$ and $\mathcal{I} \ll K^{*}$, as required. 
Next, in order to show that the map $\phi$ is an embedding, we prove that $\phi\left(\mathcal{N}_{\epsilon}(K)\right)$ is a relative Scott open, for any basic open set $\mathcal{N}_{\epsilon}(K)$. By the assumption, $K$ is $d^{-1}$-precompact. Hence there is a finite subset $\left\{k_{1}, \ldots, k_{m}\right\}$ of $K$ such that for any $k \in K, d\left(k, k_{i}\right)<\epsilon / 4$, for some $1 \leq i \leq m$. Assume that $\left(F_{n}\right)_{n>0}$ is a standard representation of $K^{*}$. Choose sufficiently large $N>0$ such that $r F_{N}<\epsilon / 4$ and at the same time for any $k_{i} \in\left\{k_{1}, \ldots, k_{m}\right\}$ there is $\left(x_{i}, r_{i}\right) \in F_{N}$ so that $d^{*}\left(k_{i}, x_{i}\right)<\epsilon / 4$. The latter property can be achieved by Lemma 3.2. Set $\mathcal{I}=\left[\left(E_{n}\right)_{n>0}\right]$, where $E_{n}=F_{N}+\frac{1}{n}$. Clearly $K^{*} \in \Uparrow \mathcal{I}$. Now, for proving

$$
\Uparrow \mathcal{I} \cap \phi\left(\mathcal{K}_{0}(X)\right) \subseteq \phi\left(\mathcal{N}_{\epsilon}(K)\right),
$$

take $B^{*} \in \Uparrow \mathcal{I}$ and show that $H_{d}(K, B)<\epsilon$. Let $k \in K$. Select $k_{i},\left(x_{i}, r_{i}\right) \in F_{N}$ and (similar to the proof of (**) in the preceding theorem) $(b, 0) \in B$ such that $d\left(k, k_{i}\right)<\epsilon / 4$, $d\left(k_{i}, x_{i}\right)<\epsilon / 4$ and $\left(x_{i}, r_{i}\right) \sqsubseteq(b, 0)$. Therefore

$$
\begin{aligned}
d(k, b) & \leq d\left(k, k_{i}\right)+d\left(k_{i}, x_{i}\right)+d\left(x_{i}, b\right) \\
& <\frac{\epsilon}{4}+\frac{\epsilon}{4}+\frac{\epsilon}{4}=\frac{3 \epsilon}{4} .
\end{aligned}
$$

In other words, $H_{d}^{-}(K, B)<\epsilon$. Next, for $H_{d}^{+}(K, B)<\epsilon$, we pick up $b \in B$. One can use the same argument used in the proof of $(*)$ in the preceding theorem to find $(x, r) \in F_{N}$ such that $d(x, b) \leq r<\epsilon / 3$. Since $x \in K, H_{d}^{+}(K, B)<\epsilon$ follows.

Roughly speaking, the above theorems state that under certain conditions on $(X, d)$ the hyperspace $\mathcal{K}_{0}(X)$ with respect to the Vietoris or Hausdorff topologies can be embedded in a suitable continuous $\omega$-dcpo. Hence, in the light of Definition 1.2, the following theorem is obtained.

Theorem 3.11. Let $(X, d)$ be a sequentially Yoneda-complete $T_{1}$ quasi-metric space. Then (1) The pair $(\mathbf{C B} X, \phi)$ gives an $\omega$-computational model for $\left(\mathcal{K}_{0}(X), \tau_{V}\right)$.

(2) If, in addition, any compact subset of $(X, d)$ is $d^{-1}$-precompact, then the pair $(\mathbf{C B} X, \phi)$ gives an $\omega$-computational model for $\left(\mathcal{K}_{0}(X), H_{d}\right)$.

Also, in the light of Fact 1.1 and Theorem 2.5, since the way-below relation of any continuous poset is approximating, the ideal completion of $(\mathbf{C B} X, \sqsubseteq)$ with the auxiliary relation « gives a computational model of $\mathcal{K}_{0}(X)$. So the following theorem is established.

Theorem 3.12. Let $(X, d)$ be a sequentially Yoneda-complete $T_{1}$ quasi-metric space. Then (1) $\left(\mathcal{K}_{0}(X), \tau_{V}\right)$ has a computational model.

(2) If, in addition, any compact subset of $(X, d)$ is $d^{-1}$-precompact, $\left(\mathcal{K}_{0}(X), H_{d}\right)$ has a computational model.

The following corollary is a trivial consequence of the above Theorems. See also [EH98, LK04, Mar04.

Corollary 3.13. Let $(X, d)$ be a complete metric space. Then the hyperspace $\left(\mathcal{K}_{0}(X), H_{d}\right)$ has a computational model.

It could be readily seen that the Vietoris and respectively Hausdorff topologies are $T_{1}$ if and only if the $\phi$-image of $\mathcal{K}_{0}(X)$ is a subset of the maximal elements of $\mathbf{C B} X$. It is known that both these topologies are not necessarily $T_{1}$. Therefore the $\phi$-image of $\mathcal{K}_{0}(X)$ may not lie in the maximal elements of $\mathbf{C B} X$. As it was noted before, if $(X, d)$ is Hausdorff (more generally $\mathrm{KC})$, these topologies are $T_{1}$.

The following examples give an application of Theorem 3.12 


\section{Example 3.14.}

(1) Let $\mathbb{R}$ be the set of real numbers and let $d$ be a $T_{1}$ quasi-metric defined on $\mathbb{R}$ by $d(x, y)=y-x$ if $x \leq y$ and $d(x, y)=1$ if $x>y$. Then the topology $\tau_{d}$ is the Sorgenfrey topology on $\mathbb{R}$ and $\mathbb{R}_{l}=(\mathbb{R}, d)$ called the Sorgenfrey line. It is easy to see that $(\mathbb{R}, d)$ is a Hausdorff (sequentially) Yoneda-complete space. It is a well-known fact that any compact subset of Sorgenfrey line is compact with respect to the usual topology of $\mathbb{R}$ and hence it is $d^{-1}$-precompact.

(2) Let $\Sigma$ be a non-empty set and $\Sigma^{\infty}$ be the set of finite and infinite sequences over $\Sigma$. Define the relation $\preceq$ on $\Sigma^{\infty}$ as

$$
x \preceq y \quad \leftrightarrow \quad x \text { is a prefix of } y .
$$

For $x, y \in \Sigma^{\infty}$, we denote the longest common prefix of $x$ and $y$ by $x \sqcap y$. Also the length of an element $x \in \Sigma^{\infty}$, is denoted by $l(x) \in \mathbb{N} \cup\{\infty\}$. Define $q_{b}: \Sigma^{\infty} \times \Sigma^{\infty} \rightarrow[0,1]$, given as:

We adopt the convention $\frac{1}{\infty}=0$.

$$
\begin{gathered}
q_{b}(x, y)=2^{-l(x)}-2^{-l(y)} \quad \text { if } x \preceq y, \\
q_{b}(x, y)=1 \quad \text { otherwise. }
\end{gathered}
$$

It is proved in [RRV08 that $\left(\Sigma^{\infty}, q_{b}\right)$ is Hausdorff and $\left(q_{b}\right)^{-1}$-right $K$-sequentially complete. Furthermore, it is quite straightforward to complete the argument in [RRV08] to prove this space is in fact sequentially Yoneda-complete.

(3) Let $d$ be the restriction of the Sorgenfrey metric defined in Example 1, to [0,1]. Put $X=[0,1]^{[0,1]}$ as the set of all continuous functions $f:[0,1] \rightarrow[0,1]$ with respect to the quasi-metric space $([0,1], d)$. For $f, g \in X$, let

$$
D(f, g)=\sup _{x \in[0,1]} d(f(x), g(x)) .
$$

Note that $D(f, g)<1$ forces $f \leq g$. Hence any Cauchy sequence $\left(f_{n}\right)_{n>0}$ should be eventually increasing. Now, for such a sequence take $f=\sup _{n \geq n_{0}} f_{n}$, where $n_{0}$ is the index from which the sequence is increasing. Then, $f$ is the Yoneda-limit of $\left(f_{n}\right)_{n>0}$ and hence $(X, D)$ is a $T_{1}$ sequentially Yoneda-complete space. In fact, it can be readily seen that $(X, D)$ is a Hausdorff space.

(4) Let $\mathcal{C}=[0,1]^{\omega}$. For $p \in[1, \infty)$ the function $q_{p}: \mathcal{C} \rightarrow[0,1]$ is defined by

$$
q_{p}(f)=\left(\sum_{n=0}^{\infty}\left(2^{-n} f(n)\right)^{p}\right)^{\frac{1}{p}} .
$$

Then using an argument in [RSV03], Theorem 1, one can show that the function $f_{q_{p}}$ : $\mathcal{C} \times \mathcal{C} \rightarrow[0,1]$ given by

$$
\begin{gathered}
f_{q_{p}}(f, g)=q_{p}(f-g) \quad \text { if } f \geq g, \\
f_{q_{p}}(f, g)=1 \quad \text { otherwise, }
\end{gathered}
$$

defines a Hausdorff quasi-metric on $\mathcal{C}$. Furthermore, by adopting the proofs of Theorems 3 and 4 in RSV03, one can also show that $\left(\mathcal{C}, f_{q_{p}}\right)$ is sequentially Yoneda-complete. (This quasi-metric is in fact the conjugate of the quasi-metric $e_{d_{p}}$ given in RSV03.)

Corollary 3.15. Let $(X, d)$ be one of the examples given in 3.14. Then the space $\left(\mathcal{K}_{0}(X), \tau_{V}\right)$ is $T_{1}$ and has a computational model. Furthermore in the case of Sorgenfrey line $\mathbb{R}_{l}$, the space $\left(\mathcal{K}_{0}\left(\mathbb{R}_{l}\right), H_{d}\right)$ is $T_{1}$ and has a computational model. 


\section{A quantitative $\omega$-Computational model of $\mathcal{K}_{0}(X)$}

So far we have shown that, under certain circumstances, $\left(\mathcal{K}_{0}(X), H_{d}\right)$ can be embedded in $\mathbf{C B} X$ and therefore $(\mathbf{C B} X, \phi)$ provides an $\omega$-computational model for $\left(\mathcal{K}_{0}(X), H_{d}\right)$. In this section, we take the well-known quantitative $(\omega$-)computational model approach, proving that $\mathbf{C B} X$ is also a quantitative $\omega$-computational model for $\left(\mathcal{K}_{0}(X), H_{d}\right)$ and so the results of the preceding section are strengthened. Our definition of quantitative $(\omega$-)computational model follows Rutten ([Rut98, Section 7).

Definition 4.1. A quantitative ( $\omega$-) computational model of a quasi-metric space $(Y, d)$ is a quadruple $(L, \sqsubseteq, D, \phi)$ where $(L, \sqsubseteq)$ is a continuous $(\omega-)$ dcpo, $D$ is an algebraic sequentially Yoneda-complete quasi-metric on $L$ and $\phi: Y \rightarrow L$ is a map such that:

(1) The specialization partial order $\sqsubseteq_{D}$ is equivalent to the partial order of $L$.

(2) $\phi$ is an isometry from $(Y, d)$ into $(L, D)$.

(3) $\phi\left(\operatorname{Max}\left(Y, \sqsubseteq_{d}\right)\right)=\operatorname{Max}(L, \sqsubseteq)$.

It is worth mentioning that the above definition is weaker than the definition of Romaguera and Valero (Definition 1 in RV09]) in which $(L, D)$ is considered to be Smythcomplete and the topology $\tau_{D}$ coincides with the Scott topology $\sigma_{L}$. We prefer to take Rutten's notion of quantitative $\omega$-computational model and then study a special case where $(L, D)$ satisfies the extra conditions of Definition 1 in [RV09] (Theorems 4.16).

In [RV09], Romaguera and Valero followed the work of Heckmann [Hec99] for a complete weighted quasi-metric space $(X, d)$ and defined a complete partial quasi-metric $Q$ on the space of formal balls. A quasi-metric $q$ on $\mathbf{B} X$ is then derived from $Q$ which induces the same topology as $Q$ on $\mathbf{B} X$ and moreover, $(\mathbf{B} X, q)$ is Smyth-complete. It is useful to note that the quasi-metric $q$ can be defined directly on $\mathbf{B} X$ for any quasi-metric space without the existence of a partial metric.

Definition 4.2. Let $(X, d)$ be a quasi-metric space. For $(x, r),(y, s) \in \mathbf{B} X$, define

$$
q((x, r),(y, s))=\max \{d(x, y),|r-s|\}+(s-r) .
$$

It is easy to see that $q$ defines a quasi-metric on $\mathbf{B} X$. The next lemma shows that $(\mathbf{B} X, q)$ inherits Smyth-completeness and sequentially Yoneda-completeness from $(X, d)$. The proof is more or less the same as the proof of Theorem 4.1 of [RV09].

Lemma 4.3. Let $(X, d)$ be a sequentially Yoneda-complete (respectively Smyth-complete) quasi-metric space. Then $(\mathbf{B} X, q)$ is also sequentially Yoneda-complete (respectively Smythcomplete).

The following theorem generalizes Theorem 5.1 of [RV09].

Theorem 4.4. Each algebraic sequentially Yoneda-complete $T_{1}$ quasi-metric space has a quantitative computational model.

Proof. Let $(X, d)$ be an algebraic sequentially Yoneda-complete $T_{1}$ quasi-metric space and $q$ be the quasi-metric defined in Definition 4.2 on $\mathbf{B} X$. By Theorem 2.2, $\mathbf{B} X$ is a continuous dcpo. Also, by the above lemma $(\mathbf{B} X, q)$ is sequentially Yoneda-complete. A straightforward computation shows that for any finite element $x \in X,(x, r)$ is finite in $(\mathbf{B} X, q)$ and moreover the set of such elements forms a base for $(\mathbf{B} X, q)$. Therefore $(\mathbf{B} X, q)$ is algebraic. The other parts follow easily from the proof of Theorem 4.1 of [RV09]. 
Now, we turn to the main topic of this section. We wish to define a quasi-metric $D$ on $\mathbf{C B} X$ and show that $(\mathbf{C B} X, D)$ together with the map $\phi: \mathcal{K}_{0}(X) \rightarrow \mathbf{C B} X$ which is defined as $\phi(K)=K^{*}$, form a quantitative computational model for $\left(\mathcal{K}_{0}(X), H_{d}\right)$.

To emphasize, we fix $(X, d)$ to be a sequentially Yoneda-complete $T_{1}$ quasi-metric space, though $(X, d)$ need not be algebraic.

Definition 4.5. Let $q$ be the quasi-metric defined in Definition 4.2 on $\mathbf{B} X$. Recall that $H_{q}$ on $\mathcal{P}_{\text {fin }} \mathbf{B} X$ is defined by

$$
H_{q}(F, G)=\max \left\{\sup _{(x, r) \in F} \inf _{(y, s) \in G} q((x, r),(y, s)), \sup _{(y, s) \in G} \inf _{(x, r) \in F} q((x, r),(y, s))\right\},
$$

for any $F, G \in \mathcal{P}_{\text {fin }} \mathbf{B} X$. Put $D$ on $\mathbf{C B} X$ as follows:

$$
D(\mathcal{I}, \mathcal{J})=\sup _{F_{n}} \inf _{G_{m}} H_{d}\left(F_{n}, G_{m}\right),
$$

for all $\mathcal{I}, \mathcal{J} \in \mathbf{C B} X$ and representations $\left(F_{n}\right)_{n>0},\left(G_{m}\right)_{m>0}$ of $\mathcal{I}$ and $\mathcal{J}$, respectively.

Next, we show that $D$ is independent from any particular choice of representations.

Lemma 4.6. $D$ is well-defined on $\mathbf{C B} X$.

Proof. Let $\left(F_{n}\right)_{n>0}$ and $\left(F_{k}^{\prime}\right)_{k>0}$ be two different representations for $\mathcal{I}$. Since $\left(F_{n}\right)_{n>0} \sim$ $\left(F_{k}^{\prime}\right)_{k>0}$, for any $F_{n}$ there is $F_{k}^{\prime}$ such that $F_{n} \prec F_{k}^{\prime}$. So $H_{q}\left(F_{n}, F_{k}^{\prime}\right)=0$ and therefore

$$
H_{q}\left(F_{n}, G_{m}\right) \leq H_{q}\left(F_{k}^{\prime}, G_{m}\right) .
$$

So by taking infimum on $G_{m}$, we have

$$
\inf _{G_{m}} H_{q}\left(F_{n}, G_{m}\right) \leq \inf _{G_{m}} H_{q}\left(F_{k}^{\prime}, G_{m}\right) .
$$

Thus

$$
\begin{gathered}
\inf _{G_{m}} H_{q}\left(F_{n}, G_{m}\right) \leq \sup _{F_{k}^{\prime}} \inf _{G_{m}} H_{q}\left(F_{k}^{\prime}, G_{m}\right), \\
\sup _{F_{n}} \inf _{G_{m}} H_{q}\left(F_{n}, G_{m}\right) \leq \sup _{F_{k}^{\prime} \inf _{m}} H_{q}\left(F_{k}^{\prime}, G_{m}\right) .
\end{gathered}
$$

Similarly, we can prove that

$$
\sup _{F_{k}^{\prime}} \inf _{G_{m}} H_{q}\left(F_{k}^{\prime}, G_{m}\right) \leq \sup _{F_{n}} \inf _{G_{m}} H_{q}\left(F_{n}, G_{m}\right) .
$$

Proposition 4.7. $D$ is a quasi-metric on $\mathbf{C B} X$. In addition, the specialization order $\sqsubseteq_{D}$ is equivalent to the partial order $\sqsubseteq$ defined on $\mathbf{C B} X$.

Proof. The triangular inequality is straightforward. So we only check that $D(\mathcal{I}, \mathcal{J})=$ $D(\mathcal{J}, \mathcal{I})=0$ implies $\mathcal{I}=\mathcal{J}$. Let $D(\mathcal{I}, \mathcal{J})=0$ and $\left(F_{n}\right)_{n>0}$ and $\left(G_{m}\right)_{m>0}$ be representations for $\mathcal{I}$ and $\mathcal{J}$, respectively. Fix $n>0$ and put $\delta=\delta\left(F_{n}, F_{n+1}\right)$. From the definition of $D$, there is $m>0$ such that $H_{q}\left(F_{n+1}, G_{m}\right)<\delta$. We prove $F_{n} \prec G_{m}$. Let $(x, r) \in F_{n}$. There is $\left(x^{\prime}, r^{\prime}\right) \in F_{n+1}$ with $(x, r) \prec\left(x^{\prime}, r^{\prime}\right)$. Also, by $H_{q}\left(F_{n+1}, G_{m}\right)<\delta$, there is $(y, s) \in G_{m}$ such that $q\left(\left(x^{\prime}, r^{\prime}\right),(y, s)\right)<\delta$. This means that $d\left(x^{\prime}, y\right)<r^{\prime}-s+\delta$ and consequently

$$
\begin{aligned}
d(x, y) & \leq d\left(x, x^{\prime}\right)+d\left(x^{\prime}, y\right) \\
& <\left(r-r^{\prime}\right)-\delta+\left(r^{\prime}-s\right)+\delta \\
& =r-s .
\end{aligned}
$$


Thus $(x, r) \prec(y, s)$ and therefore $F \prec_{L} G . \quad F \prec_{U} G$ can be shown in a similar fashion. Hence $F \prec G$ is established and therefore $\mathcal{I} \sqsubseteq \mathcal{J}$. Now $D(\mathcal{J}, \mathcal{I})=0$ also implies that $\mathcal{J} \sqsubseteq \mathcal{I}$.

For the second part, clearly by the above argument, $D(\mathcal{I}, \mathcal{J})=0$ implies $\mathcal{I} \sqsubseteq \mathcal{J}$. Conversely, let $\mathcal{I} \sqsubseteq \mathcal{J}$. So for any $F \in \mathcal{I}$, there is $G \in \mathcal{J}$ with $F \prec G$. Thus $H_{q}(F, G)=0$ which implies $D(\mathcal{I}, \mathcal{J})=0$.

Proposition 4.8. The domain $\mathbf{C B} X$ equipped with the quasi-metric $D$ is sequentially Yoneda-complete.

Proof. Let $\left(\mathcal{I}_{n}\right)_{n>0}$ be a Cauchy sequence in $(\mathbf{C B} X, D)$ and for any $n>0,\left(F_{m}^{n}\right)_{m>0}$ be a representation for $\mathcal{I}_{n}$. For any $n>0$, there is a natural number $N_{n}>n$ such that $N_{n}>N_{n-1}$ for $n>1$ and for any $l \geq k \geq N_{n}, D\left(\mathcal{I}_{k}, \mathcal{I}_{l}\right)<\frac{1}{2^{n+1}}$, i.e.

$$
\forall F_{r}^{k} \in \mathcal{I}_{k} \quad \exists F_{s}^{l} \in \mathcal{I}_{l} \quad H_{q}\left(F_{r}^{k}, F_{s}^{l}\right)<\frac{1}{2^{n+1}} .
$$

Define $\left(G_{i j}\right)_{i, j>0}$ as follows:

Fix $F_{1}^{N_{1}} \in \mathcal{I}_{N_{1}}$ and put $G_{11}=F_{1}^{N_{1}}$. Inductively for any $k \geq 2$, choose $G_{1 k} \in\left(F_{m}^{N_{k}}\right)_{m>0}$ such that

$$
H_{q}\left(G_{1 k-1}, G_{1 k}\right)<\frac{1}{2^{k}}
$$

In a similar way, for any $i \geq 2$, put $G_{i 1}=F_{i}^{N_{1}}$ and inductively find $\left(G_{i k}\right)_{k>0}$ such that for any $i<j, G_{i k} \prec G_{j k}$ and

$$
H_{q}\left(G_{i k-1}, G_{i k}\right)<\frac{1}{2^{k}}
$$

Let $L_{k}=G_{k k}+\frac{1}{2^{k-1}}$. The following two claims complete the proof.

Claim 1. $\mathcal{I}=\left[\left(L_{k}\right)_{k>0}\right] \in \mathbf{C B} X$, i.e. $\left(L_{k}\right)_{k>0}$ is an ascending sequence in $\mathcal{P}_{\text {fin }} \mathbf{B} X$. Let $k>0$ and $\left(x, r+\frac{1}{2^{k-1}}\right) \in L_{k}$. Hence $(x, r) \in G_{k k}$. Since

$$
\begin{aligned}
H_{q}\left(G_{k k}, G_{k+1 k+1}\right) & \leq H_{q}\left(G_{k k}, G_{k+1 k}\right)+H_{q}\left(G_{k+1 k}, G_{k+1 k+1}\right) \\
& <0+\frac{1}{2^{k+1}}
\end{aligned}
$$

there is a $(y, s) \in G_{k+1 k+1}$ such that $q((x, r),(y, s))<\frac{1}{2^{k+1}}$. Hence $d(x, y)<r-s+\frac{1}{2^{k+1}}$. By a simple calculation,

$$
d(x, y)<\left(r+\frac{1}{2^{k-1}}\right)-\left(s+\frac{1}{2^{k}}\right) .
$$

Therefore $\left(x, r+\frac{1}{2^{k-1}}\right) \prec\left(y, s+\frac{1}{2^{k}}\right)$, where $\left(y, s+\frac{1}{2^{k}}\right) \in L_{k+1}$. This means that $L_{k} \prec{ }_{L} L_{k+1}$. Similarly, we can prove $L_{k} \prec_{U} L_{k+1}$ and consequently $L_{k} \prec L_{k+1}$.

Claim 2. $\mathcal{I}$ is the Yoneda limit of $\left(\mathcal{I}_{n}\right)_{n>0}$.

First, we prove that $D\left(\mathcal{I}_{n}, \mathcal{I}\right) \rightarrow 0$. Let $\epsilon>0$. Since $\left(\mathcal{I}_{n}\right)_{n>0}$ is a Cauchy sequence, there is $N>0$ such that for any $r \geq s \geq N, D\left(\mathcal{I}_{s}, \mathcal{I}_{r}\right)<\frac{\epsilon}{3}$. Fix $n>N$ and $F \in \mathcal{I}_{n}=\left[\left(F_{m}^{n}\right)_{m>0}\right]$. Choose $N_{k}>n$ from the cofinal sequence $\left(N_{n}\right)_{n>0}$ constructed above such that $\frac{1}{2^{k-1}}<\frac{\epsilon}{3}$. Since $D\left(\mathcal{I}_{n}, \mathcal{I}_{N_{k}}\right)<\frac{\epsilon}{3}$, there is $F_{m_{0}}^{N_{k}} \in \mathcal{I}_{N_{k}}$ such that $H_{q}\left(F, F_{m_{0}}^{N_{k}}\right)<\frac{\epsilon}{3}$. Choose $G_{l k} \in \mathcal{I}_{N_{k}}$ with $F_{m_{0}}^{N_{k}} \prec G_{l k}$ and $l>k$. Now, $H_{q}\left(G_{l k}, G_{l l}\right)<\frac{1}{2^{k+1}}+\cdots+\frac{1}{2^{l}}<\frac{1}{2^{k}}<\frac{\epsilon}{3}$ implies that

$$
\begin{aligned}
H_{q}\left(F, L_{l}\right) & \leq H_{q}\left(F, F_{m_{0}}^{N_{k}}\right)+H_{q}\left(F_{m_{0}}^{N_{k}}, G_{l k}\right)+H_{q}\left(G_{l k}, G_{l l}\right)+H_{q}\left(G_{l l}, L_{l}\right) \\
& <\frac{\epsilon}{3}+0+\frac{\epsilon}{3}+\frac{\epsilon}{3} \\
& =\epsilon
\end{aligned}
$$


Now, we show that $\mathcal{I}$ is the Yoneda limit of $\left(\mathcal{I}_{n}\right)_{n>0}$. Because of $D\left(\mathcal{I}_{n}, \mathcal{I}\right) \rightarrow 0$, it suffices to prove that $D(\mathcal{I}, \mathcal{J}) \leq \inf _{n} \sup _{k>n} D\left(\mathcal{I}_{k}, \mathcal{J}\right)$, for any $\mathcal{J} \in \mathbf{C B} X$. Put $\inf _{n} \sup _{k>n} D\left(\mathcal{I}_{k}, \mathcal{J}\right)=$ $s$. We prove that for any $\epsilon>0, D(\mathcal{I}, \mathcal{J}) \leq s+\epsilon$. Let $L \in \mathcal{I}$. Since $\inf _{n} \sup _{k>n} D\left(\mathcal{I}_{k}, \mathcal{J}\right)=s$, there is $N>0$ such that for any $n>N, D\left(\mathcal{I}_{n}, \mathcal{J}\right) \leq s+\epsilon / 2$. Take $k>0$ such that $L \prec L_{k}$, $\frac{1}{2^{k-1}}<\epsilon / 2$ and $N_{k}>N$. So, for any representation $\left(G_{m}\right)_{m>0}$ of $\mathcal{J}$, there is $m>0$ such that

$$
\begin{aligned}
H_{q}\left(L, G_{m}\right) & \leq H_{q}\left(L, L_{k}\right)+H_{q}\left(L_{k}, G_{k k}\right)+H_{q}\left(G_{k k}, G_{m}\right) \\
& <0+\frac{1}{2^{k-1}}+s+\epsilon / 2 \\
& <s+\epsilon
\end{aligned}
$$

This complete the proof.

Below, we show that the quasi-metric space $(\mathbf{C B} X, D)$ is in fact the sequential Yoneda completion of $\left(\mathcal{P}_{\text {fin }} \mathbf{B} X, H_{d}\right)$. First, we recall the definition of sequential Yoneda completion KS02].

Definition 4.9. Let $(Y, d)$ be a quasi-metric space and

$$
\widehat{Y}=\left\{\left(x_{n}\right)_{n>0}:\left(x_{n}\right)_{n>0} \text { is a Cauchy sequence }\right\} .
$$

Define the quasi-pseudometric $\widehat{d}$ and the equivalence relation $\approx$ as follows:

$$
\begin{gathered}
\widehat{d}\left(\left(x_{n}\right)_{n>0},\left(y_{m}\right)_{m>0}\right)=\inf _{n} \sup _{k \geq n} \sup _{m \geq m} \inf _{p \geq m} d\left(x_{k}, y_{p}\right), \\
\left(x_{n}\right)_{n>0} \approx\left(y_{m}\right)_{m>0} \Leftrightarrow \widehat{d}\left(\left(x_{n}\right)_{n>0},\left(y_{m}\right)_{m>0}\right)=\widehat{d}\left(\left(y_{m}\right)_{m>0},\left(x_{n}\right)_{n>0}\right)=0 .
\end{gathered}
$$

Put $\bar{Y}=\widehat{Y} / \approx$ and define the quasi-metric $\bar{d}$ by

$$
\bar{d}\left(\left[\left(x_{n}\right)_{n>0}\right],\left[\left(y_{m}\right)_{m>0}\right]\right)=\widehat{d}\left(\left(x_{n}\right)_{n>0},\left(y_{m}\right)_{m>0}\right) .
$$

The pair $(\bar{Y}, \bar{d})$ is called the sequential Yoneda completion of $(Y, d)$.

Proposition 4.10. $(\mathbf{C B} X, D)$ is the sequential Yoneda completion of $\left(\mathcal{P}_{\text {fin }} \mathbf{B} X, H_{d}\right)$.

Proof. Let $\left(F_{n}\right)_{n>0}$ be a Cauchy sequence in $\mathcal{P}_{\text {fin }} \mathbf{B} X$ and $\bar{H}_{d}$ be the quasi-metric completion of $H_{d}$ (Definition 4.9). Without loss of generality, we can assume that for any $0<n \leq m$,


property. Now, we show that there is an ascending chain $\left(L_{k}\right)_{k>0}$ in $\mathcal{P}_{\text {fin }} \mathbf{B} X$ which is

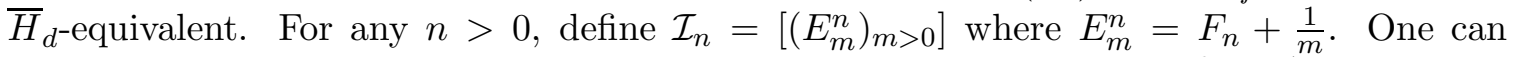
readily check that $\left(\mathcal{I}_{n}\right)_{n>0}$ is a Cauchy sequence. For $k>0$, put $L_{k}=E_{k}^{k}+\frac{1}{2^{k-1}}$. A similar argument as used in Claim 1 of Proposition 4.8 , shows that $\left[\left(L_{k}\right)_{k>0}\right]$ is a $\prec$-ascending chain. In order to show

$$
\bar{H}_{d}\left(\left(F_{n}\right)_{n>0},\left(L_{m}\right)_{m>0}\right)=0,
$$

we have to verify the following:

$$
\forall \epsilon>0 \exists n \forall k \geq n \forall m \exists p \geq m H_{d}\left(F_{k}, L_{p}\right)<\epsilon .
$$

Let $\epsilon>0$ be given. Find $n>0$ so that $\frac{1}{2^{n+1}}<\frac{\epsilon}{2}$. Next, for any $k \geq n$ and $m>0$, take $p \geq k, m$ such that $\frac{1}{p}+\frac{1}{2^{p-1}}<\frac{\epsilon}{2}$. Since $L_{p}=F_{p}+\left(\frac{1}{p}+\frac{1}{2^{p-1}}\right)$, it easily follows that $H_{d}\left(F_{k}, L_{p}\right)<\epsilon$. The equality $\bar{H}_{d}\left(\left(L_{m}\right)_{m>0},\left(F_{n}\right)_{n>0}\right)=0$ can be proved in a similar way.

So, the map

$$
\begin{array}{rlll}
\psi: & \left(\overline{\mathcal{P}_{f i n} \mathbf{B} X}, \bar{H}_{d}\right) & \longrightarrow & (\mathbf{C B} X, D) \\
{\left[\left(F_{n}\right)_{n>0}\right]} & \longmapsto & {\left[\left(L_{k}\right)_{k>0}\right] .}
\end{array}
$$

defines a bijective isometry, as required. 
Corollary 4.11. The domain $\mathbf{C B} X$ equipped with the quasi-metric $D$ is algebraic.

Proof. By Corollary 23 of [KS02, any Yoneda completion is algebraic. Hence, according to Proposition 4.10, $(\mathbf{C B} X, D)$ is algebraic.

Proposition 4.12. The map $\phi$ is an isometry between $\left(\mathcal{K}_{0}(X), H_{d}\right)$ and $(\mathbf{C B} X, D)$.

Proof. Let $K_{1}, K_{2} \in \mathcal{K}_{0}(X)$ and $\left(F_{n}\right)_{n>0}$ and $\left(G_{m}\right)_{m>0}$ be standard representations for $K_{1}$ and $K_{2}$, respectively.

Assume that $H_{d}\left(K_{1}, K_{2}\right)=d$ and $\epsilon>0$. We show that $D\left(K_{1}^{*}, K_{2}^{*}\right) \leq d+\epsilon$. Fix $n>0$ and let $\delta=\delta\left(F_{n}, F_{n+1}\right)$. We wish to find $m>0$ such that

$$
H_{q}\left(F_{n}, G_{M}\right)<d+\epsilon .
$$

Let $(x, r) \in F_{n}$. Since $F_{n} \prec F_{n+1} \prec K_{1}$, there are $\left(x^{\prime}, r^{\prime}\right) \in F_{n+1}$ and $(z, 0) \in K_{1}$ such that $d\left(x, x^{\prime}\right)<\left(r-r^{\prime}\right)-\delta$ and $d\left(x^{\prime}, z\right)<r^{\prime}$. Because of $H_{d}\left(K_{1}, K_{2}\right)=d$, there is $y \in K_{2}$ such that $d(z, y) \leq d+\epsilon$. By Lemma 3.2, there is a sequence $\left(\left(y_{m}, s_{m}\right)\right)_{m>0}$ such that $\left(y_{m}, s_{m}\right) \in G_{m}$ and $d^{*}\left(y_{m}, y\right) \rightarrow 0$. Select $y_{m}$ such that $d\left(y, y_{m}\right)<\frac{\delta}{2}$ and $s_{m}<\frac{\delta}{2}$. Then

$$
\begin{aligned}
d\left(x, y_{m}\right) & \leq d\left(x, x^{\prime}\right)+d\left(x^{\prime}, z\right)+d(z, y)+d\left(y, y_{m}\right) \\
& <\left(r-r^{\prime}\right)-\delta+r^{\prime}+d+\epsilon+\frac{\delta}{2} \\
& =r+d+\epsilon-\frac{\delta}{2}
\end{aligned}
$$

Thus for any $(x, r) \in F_{n}$, there is $\left(y_{m_{x}}, s_{m_{x}}\right) \in G_{m_{x}}$ such that $d\left(x, y_{m_{x}}\right)<r-s_{m_{x}}+d+\epsilon$. Take $M \geq \max \left\{m_{x}:(x, r) \in F_{n}\right\}$ with $r G_{M}<\frac{\delta}{2}$. Hence, by the above calculation, for any $(x, r) \in F_{n}$ there exists $(y, s) \in G_{M}$ such that $q((x, r),(y, s))<d+\epsilon$. Similarly, for any $(y, s) \in G_{M}$ one can find $(x, r) \in F_{n}$ with

$$
q((x, r),(y, s))<d+\epsilon .
$$

So $H_{q}\left(F_{n}, G_{M}\right)<d+\epsilon$ and therefore $D\left(K_{1}^{*}, K_{2}^{*}\right) \leq H_{d}\left(K_{1}, K_{2}\right)$.

On the other hand, we show that $H_{d}\left(K_{1}, K_{2}\right) \leq D\left(K_{1}^{*}, K_{2}^{*}\right)$. Let $D\left(K_{1}^{*}, K_{2}^{*}\right)=d$ and $x \in K_{1}$. Again, Lemma 3.2 implies the existence of a sequence $\left(\left(x_{n}, r_{n}\right)\right)_{n>0}$ such that $\left(x_{n}, r_{n}\right) \in F_{n}$ and $d^{*}\left(x_{n}, x\right) \rightarrow 0$. For $\epsilon>0$, choose $\left(x_{N}, r_{N}\right)$ such that $d\left(x, x_{N}\right)<\frac{\epsilon}{3}$ and $r_{N}<\frac{\epsilon}{3}$. Since $D\left(K_{1}^{*}, K_{2}^{*}\right)=d$, there is $G_{m_{N}}$ and $\left(y_{m_{N}}, s_{m_{N}}\right) \in G_{m_{N}}$ such that $d\left(x_{N}, y_{m_{N}}\right)<r_{N}-s_{m_{N}}+d+\frac{\epsilon}{3}$. The following inequalities

$$
\begin{aligned}
d\left(x, y_{m_{N}}\right) & \leq d\left(x, x_{N}\right)+d\left(x_{N}, y_{m_{N}}\right) \\
& <\frac{\epsilon}{3}+r_{N}-s_{m_{N}}+d+\frac{\epsilon}{3} \\
& <d+\epsilon,
\end{aligned}
$$

imply $\inf _{y \in K_{2}} d(x, y) \leq d$ and therefore

$$
\sup _{x \in K_{1}} \inf _{y \in K_{2}} d(x, y) \leq d .
$$

Now, in a similar way, $\sup _{y \in K_{2}} \inf _{x \in K_{1}} d(x, y) \leq d$. Hence $H_{d}\left(K_{1}, K_{2}\right) \leq d$ and consequently $H_{d}\left(K_{1}, K_{2}\right) \leq D\left(K_{1}^{*}, K_{2}^{*}\right)$. 
Now, the main theorem of this section is stated.

Theorem 4.13. $(\mathbf{C B} X, D)$ together with the map $\phi: \mathcal{K}_{0}(X) \rightarrow \mathbf{C B} X$ form a quantitative $\omega$-computational model for $\left(\mathcal{K}_{0}(X), H_{d}\right)$.

Proof. By the above propositions, $(\mathbf{C B} X, D)$ is an algebraic sequentially Yoneda-complete quasi-metric space, the specialization partial order $\sqsubseteq_{D}$ is equivalent to the partial order of $\mathbf{C B} X$ and the map $\phi$ is an isometry between $\left(\mathcal{K}_{0}(X), H_{d}\right)$ and $(\mathbf{C B} X, D)$. The condition $\phi\left(\operatorname{Max}\left(\mathcal{K}_{0}(X), \sqsubseteq_{H_{d}}\right)\right)=\operatorname{Max}(\mathbf{C B X}, \sqsubseteq)$ follows from propositions 3.8 and 4.7 .

Next, we impose some extra conditions on $(X, d)$ under which this space has a quantitative $\omega$-computational model in the sense of Romaguera and Valero [RV09]. We, hereby, suppose that $(X, d)$ is Smyth-complete and any compact subset of $(X, d)$ is $d^{-1}$-precompact. These conditions guarantee that any Cauchy sequence in $\left(\mathcal{P}_{\text {fin }} \mathbf{B} X, H_{q}\right)$ is biCauchy.

Lemma 4.14. Let $(X, d)$ be a Smyth-complete quasi-metric space of which all of its compact subsets are $d^{-1}$-precompact. Then any $\omega$-chain in $\mathcal{P}_{\text {fin }} \mathbf{B} X$ is biCauchy.

Proof. First note, one can easily show that any compact subset of $(\mathbf{B} X, q)$ is $q^{-1}$-precompact. Now, let $\left(F_{n}\right)_{n>0}$ be an $\omega$-chain in $\mathcal{P}_{\text {fin }} \mathbf{B} X$. For any $n<m, F_{n} \prec F_{m}$. Hence $H_{q}\left(F_{n}, F_{m}\right)=$ 0 and $\left(F_{n}\right)_{n>0}$ is a Cauchy sequence. According to Lemma 4.3. $(\mathbf{B} X, q)$ is Smyth-complete. So by Theorem 1.3. $\left(\mathcal{K}_{0}(\mathbf{B} X), H_{q}\right)$ is Smyth-complete. Therefore $\mathcal{P}_{\text {fin }} \mathbf{B} X$ as a subspace of $\mathcal{K}_{0}(\mathbf{B} X)$ is Smyth-completable and so any Cauchy sequence in $\left(\mathcal{P}_{\text {fin }} \mathbf{B} X, H_{q}\right)$ is biCauchy. Hence $\left(F_{n}\right)_{n>0}$ is a biCauchy sequence.

The following auxiliary lemma will be useful in several proofs.

Lemma 4.15. Let $G \prec H \sqsubseteq M$ and $H_{q}(M, L)<\delta(G, H)$. Then $G \prec L$.

Proof. Let $(x, r) \in G$ and $\delta=\delta(G, H)$. Then there are $(y, s) \in H,(z, t) \in M$ and $(a, u) \in L$ such that

$$
(x, r) \prec(y, s) \sqsubseteq(z, t) \text { and } q((z, t),(a, u))<\delta .
$$

Then the following inequalities follow:

$$
\begin{aligned}
d(x, a) & \leq d(x, y)+d(y, z)+d(z, a) \\
& <(r-s)-\delta+(s-t)+(t-u)+\delta \\
& =r-u .
\end{aligned}
$$

Hence $(x, r) \prec(a, u)$ and consequently $G \prec_{L} L$. A similar calculation shows that $G \prec_{U} L$ and therefore $G \prec L$ as required.

Theorem 4.16. Under the assumptions of Lemma 4.14,

(1) $(\mathbf{C B} X, D)$ is Smyth-complete.

(2) The topology $\tau_{D}$ induced by $D$ coincides with the Scott topology of the domain $\mathbf{C B} X$.

Proof. (1) Let $\left(\mathcal{I}_{n}\right)_{n>0}$ be a Cauchy sequence in $(\mathbf{C B} X, D)$ and for any $n>0,\left(F_{m}^{n}\right)_{m>0}$ be a representation for $\mathcal{I}_{n}$. Now, fix the natural sequence $\left(N_{n}\right)_{n>0}$, the double sequence $\left(G_{i j}\right)_{i, j>0}$ and $\mathcal{I}=\left[\left(L_{k}\right)_{k>0}\right]$ which are constructed in the proof of Proposition 4.8. We show that $\left(\mathcal{I}_{n}\right)_{n>0}$ converges strongly to $\mathcal{I}$. Because of $D\left(\mathcal{I}_{n}, \mathcal{I}\right) \rightarrow 0$, it suffices to verify $D\left(\mathcal{I}, \mathcal{I}_{n}\right) \rightarrow 0$. Let $\epsilon>0$. Since $\left(L_{k}\right)_{k>0}$ is a Cauchy $\omega$-chain in $\left(\mathcal{P}_{\text {fin }} \mathbf{B} X, H_{q}\right)$, so, by Lemma 4.14, it is a biCauchy sequence. Choose $k>0$ such that $\frac{1}{2^{k-1}}<\epsilon / 4$ and for any $r, s \geq k, H_{q}\left(L_{s}, L_{r}\right)<\epsilon / 4$. We show that for any $n>N_{k}, D\left(\mathcal{I}, \mathcal{I}_{n}\right)<\epsilon$. 
Take $n>N_{k}$ and $L_{i} \in \mathcal{I}$. Recall that the sequence $\left(N_{j}\right)_{j>0}$ has the following properties:

$$
\forall r \geq s \geq N_{j}, \quad D\left(\mathcal{I}_{s}, \mathcal{I}_{r}\right)<\frac{1}{2^{j+1}} .
$$

Particularly $D\left(\mathcal{I}_{N_{k}}, \mathcal{I}_{n}\right)<\frac{1}{2^{k+1}}$. So, there is $F_{m}^{n} \in \mathcal{I}_{n}$ such that for $G_{k k} \in \mathcal{I}_{N_{k}}, H_{q}\left(G_{k k}, F_{m}^{n}\right)<$ $\epsilon / 4$. Now, if $i \leq k$, then $H_{q}\left(L_{i}, L_{k}\right)=0$. Otherwise, since $\left(L_{k}\right)_{k>0}$ is a biCauchy sequence, $H_{q}\left(L_{i}, L_{k}\right)<\frac{\epsilon}{4}$. Thus

$$
\begin{aligned}
H_{q}\left(L_{i}, F_{m}^{n}\right) & \leq H_{q}\left(L_{i}, L_{k}\right)+H_{q}\left(L_{k}, G_{k k}\right)+H_{q}\left(G_{k k}, F_{m}^{n}\right) \\
& <\frac{\epsilon}{4}+\frac{1}{2^{k-1}}+\frac{\epsilon}{4} \\
& <\frac{3 \epsilon}{4}
\end{aligned}
$$

(2) Suppose that $\Uparrow \mathcal{I}$ is a basic open subset of $\mathbf{C B} X$ and $\mathcal{J} \in \Uparrow \mathcal{I}$. Let $\left(G_{n}\right)_{n>0}$ be a representation of $\mathcal{J}$. So, there is $n>0$ such that $F \prec G_{n}$, for any $F \in \mathcal{I}$. Set $\delta=$ $\delta\left(G_{n}, G_{n+1}\right)$. We prove that

$$
\mathcal{N}_{\delta}(\mathcal{J})=\{\mathcal{L}: D(\mathcal{J}, \mathcal{L})<\delta\} \subseteq \Uparrow \mathcal{I}
$$

Let $\mathcal{L} \in \mathcal{N}_{\delta}(\mathcal{J})$ and $\left(L_{m}\right)_{m>0}$ be one of its representations. From $D(\mathcal{J}, \mathcal{L})<\delta$, there is $L_{m}$ such that $H_{q}\left(G_{n+1}, L_{m}\right)<\delta$. Also, according to the Lemma 4.15, $G_{n} \prec G_{n+1} \sqsubseteq G_{n+1}$ and $H_{q}\left(G_{n+1}, L_{m}\right)<\delta$ implies that $G_{n} \prec L_{m}$. Finally, since for every $F \in \mathcal{I}, F \prec G_{n}$ it follows that for any $F \in \mathcal{I}, F \prec L_{m}$. Therefore $\mathcal{L} \in \Uparrow \mathcal{I}$.

Now, let $\mathcal{N}_{\delta}(\mathcal{I})$ be a basic open subset in $\tau_{D}$. Assume that $\left(F_{n}\right)_{n>0}$ is a representation of $\mathcal{I}$. According to Lemma 4.14, $\left(F_{n}\right)_{n>0}$ is a biCauchy sequence. So there is $N>0$ such that

$$
\forall n, m \geq N \quad H_{q}\left(F_{n}, F_{m}\right)<\frac{\delta}{2} .
$$

Put $\mathcal{J}=\left(G_{n}\right)_{n>0}$, where $G_{n}=F_{N}+\frac{1}{n}$. Clearly $\mathcal{J} \ll \mathcal{I}$. We prove that

$$
\Uparrow \mathcal{J} \subseteq \mathcal{N}_{\delta}(\mathcal{I})
$$

Let $\mathcal{L} \in \Uparrow \mathcal{J}$ and $\left(L_{m}\right)_{m>0}$ be a representation for $\mathcal{L}$. Since $\mathcal{J} \ll \mathcal{L}$, there is $L_{m}$ such that for any $n>0, G_{n} \prec L_{m}$. For any $F_{n} \in \mathcal{I}$, if $n<N$ then $H_{q}\left(F_{n}, F_{N}\right)=0$. Otherwise $H_{q}\left(F_{n}, F_{N}\right)<\frac{\delta}{2}$. Take $k>0$ with $\frac{1}{k}<\frac{\delta}{4}$. Then we have

$$
\begin{aligned}
H_{q}\left(F_{n}, L_{m}\right) & \leq H_{q}\left(F_{n}, F_{N}\right)+H_{q}\left(F_{N}, G_{k}\right)+H_{q}\left(G_{k}, L_{m}\right) \\
& <\frac{\delta}{2}+\frac{1}{k}+0 \\
& <\frac{3 \delta}{4} .
\end{aligned}
$$

Thus $D(\mathcal{I}, \mathcal{L})<\delta$ or equivalently $\mathcal{L} \in \mathcal{N}_{\delta}(\mathcal{I})$.

Remark 4.17. The isometry of $\phi$ (Proposition 4.12) and the above Theorem, imply that for any Smyth-complete $T_{1}$ quasi-metric space whose compact subsets are $d^{-1}$-precompact, the map $\phi$ is an embedding. This result was already proved in Theorem 3.10 .

\section{Plotkin Powerdomain vs. $\omega$-Plotkin domain}

In this section, we compare the Plotkin Powerdomain and $\omega$-Plokin constructions of $\mathbf{B} X$. The Plotkin powerdomain of $\mathbf{B} X$, denoted by $\mathcal{P} \mathbf{B} X$, is the ideal completion of the abstract basis $\left(\mathcal{P}_{f i n} \mathbf{B} X, \prec_{E M}\right)$. In the following, we show that for any $T_{1}$ quasi-metric space $(X, d)$ if $(X, d)$ is either Smyth-complete and all of its compact subsets are $d^{-1}$-precompact, or $\omega$-algebraic Yoneda-complete, then $\mathcal{P} \mathbf{B} X$ and $\mathbf{C B} X$ are order-isomorphic. 
Theorem 5.1. Let $(X, d)$ be a $T_{1}$ quasi-metric space. Assume also that either of the following conditions hold.

(1) $(X, d)$ is a Smyth-complete space all of whose compact subsets are $d^{-1}$-precompact.

(2) $(X, d)$ is an $\omega$-algebraic Yoneda-complete space.

Then $\mathcal{P} \mathbf{B} X$ and $\mathbf{C B} X$ are order-isomorphic.

Proof. (1) Assume first that $(X, d)$ satisfies condition 1 above. Let $\mathcal{I}$ be a round ideal in $\mathcal{P}_{\text {fin }} \mathbf{B} X$. We claim that $\mathcal{I}$ has a cofinal $\prec_{E M}$-ascending subsequence $\left(F_{n}\right)_{n>0}$. Note that $\mathcal{I}$ can be considered as a Cauchy net in $\left(\mathcal{P}_{\text {fin }} \mathbf{B} X, H_{q}\right)$, since if $G \prec_{E M} H \in \mathcal{I}$, then $H_{q}(G, H)=0$. Under the above assumptions, similar to the argument used in Lemma 4.14, $\left(\mathcal{P}_{\text {fin }} \mathbf{B} X, H_{q}\right)$ is Smyth-completable. Hence $\mathcal{I}$ is biCauchy and has a biCauchy subsequence $\left(F_{n}\right)_{n>0}$, satisfying the following property:

$$
\forall H, G \in \mathcal{I}, F_{n} \prec_{E M} H, G \quad \Rightarrow \quad H_{q}(H, G)<\frac{1}{n} .
$$

Without loss of generality, we may assume that $\left(F_{n}\right)_{n>0}$ is $\prec_{E M}$-ascending. Now, we show that this sequence is $\prec_{E M}$-cofinal in $\mathcal{I}$. Let $G \in \mathcal{I}$. $N>0$ must be found such that $G \prec_{E M} F_{N}$. Choose $H \in \mathcal{I}$ with $G \prec_{E M} H$ and put $\delta=\delta(G, H)$. Take $N>1$ such that $\frac{1}{N-1}<\delta$ and let $M \in \mathcal{I}$ with $F_{N}, H \prec_{E M} M$. Therefore by $F_{N-1} \prec_{E M} F_{N} \prec_{E M} M$ and (*), we have $H_{q}\left(M, F_{N}\right)<\frac{1}{N-1}<\delta$. Finally, according to the Lemma 4.15, $G \prec_{E M} H \prec_{E M} M$ and $H_{q}\left(M, F_{N}\right)<\delta$ implies $G \prec_{E M} F_{N}$.

Now, it is easy to check that the mapping

$$
\begin{array}{lll}
\psi: \mathcal{P} \mathbf{B} X & \longrightarrow & \mathbf{C B} X \\
\mathcal{I} & \longmapsto & {\left[\left(F_{n}\right)_{n>0}\right]}
\end{array}
$$

defines an order isomorphism.

(2) Now consider that $(X, d)$ is an $\omega$-algebraic Yoneda-complete space. Let $X_{0}$ be a countable algebraic subset of $X$. Then the set $\mathbf{B}_{\mathbb{Q}} X_{0}=X_{0} \times \mathbb{Q}^{+}$and respectively the set $\mathcal{P}_{\text {fin }} \mathbf{B}_{\mathbb{Q}} X_{0}$ of all finite subsets of $\mathbf{B}_{\mathbb{Q}} X_{0}$ form a countable basis for $\mathbf{B} X$ and for $\mathcal{P}_{\text {fin }} \mathbf{B} X$ respectively. Now, in the light of Theorem 6.2.3 of AJ94, $\mathcal{P} \mathbf{B} X$ is given by the ideal completion of $\left(\mathcal{P}_{\text {fin }} \mathbf{B}_{\mathbb{Q}} X_{0}, \prec_{E M}\right)$. Furthermore, since the set $\mathcal{P}_{\text {fin }} \mathbf{B}_{\mathbb{Q}} X_{0}$ is countable, by Proposition 2.2.3 in [AJ94], every round ideal in $\mathcal{P}_{\text {fin }} \mathbf{B}_{\mathbb{Q}} X_{0}$ has a cofinal $\prec_{E M^{-}}$ ascending subsequence and hence following the same proof as in (1), one can prove that $\left(\mathcal{P} \mathbf{B}_{\mathbb{Q}} X_{0}, \prec_{E M}\right)$ is order-isomorphic to $\left(\mathbf{C B}_{\mathbb{Q}} X_{0}, \prec_{E M}\right)$. On the other hand, one can easily show that any $\prec_{E M}$-ascending subsequence $\left(F_{n}\right)_{n>0}$ in $\mathcal{P}_{f i n} \mathbf{B} X$ is $\sim_{E M}$-equivalent to a $\prec_{E M}$-ascending subsequence $\left(G_{n}\right)_{n>0}$ in $\mathcal{P}_{\text {fin }} \mathbf{B}_{\mathbb{Q}} X_{0}$. Hence $\left(\mathbf{C B}_{\mathbb{Q}} X_{0}, \prec_{E M}\right)$ is orderisomorphic to $\left(\mathbf{C B} X, \prec_{E M}\right)$. Therefore, the proof is established.

Corollary 5.2. Let $(X, d)$ be a complete metric space. Then the Plotkin powerdomain $\mathcal{P B} X$ and $\omega$-Plotkin $\mathbf{C B} X$ are isomorphic.

\section{Future Work}

In this paper, various computational models of the hyperspace $\mathcal{K}_{0}(X)$ of the non-empty compact subsets of a quasi-metric space $(X, d)$ were studied. It was shown how to use a special computational model $\mathbf{B} X$ of $(X, d)$ to get the corresponding $\omega$-computational model $\mathbf{C B} X$ of $\mathcal{K}_{0}(X)$. The above construction would have been more satisfactory if a computational model for the $\mathcal{K}_{0}(X)$ could have been defined, starting from an arbitrary 
computational model of $(X, d)$. This idea is already developed for the case of metric spaces by Martin [Mar04, by appealing to the notion of a measurement, and for the spaces with countable based models by Berger et. al. in Ber10. Therefore, an interesting subject of research is to find a fairly general framework under which the ideas from [Mar04 and [Ber10] can be generalized to the present context. Another topic of research is to study the effectiveness of $\mathcal{K}_{0}(X)$. So one could ask whether $\mathbf{C B} X$ supports an effective base whenever $(X, d)$ is an effective quasi-metric.

Section 5 involves a generalization of the results obtained earlier, showing that $\mathcal{K}_{0}(X)$ has a quantitative $\omega$-computational model. One would desire to have a quantitative computational model for $\mathcal{K}_{0}(X)$. This, for example, requires to generate another computational model from $(\mathbf{C B} X, \sqsubseteq, D, \phi)$, similar to what we obtained in Theorem 3.12. One way to achieve this is to employ the Yoneda-completion of $(\mathbf{C B} X, D)$, which serves as a natural generalization of the ideal completion.

In section 6 , the Plotkin powerdomain and $\omega$-Plotkin domain of $\mathbf{B} X$ were compared and the situations in which both constructions are order-isomorphic were observed. Now, the question of finding an example for which these constructions are not isomorphic is imposed.

\section{ACKNOWLEDGEMENT}

The authors would like to thank R. Heckmann and O. Valero for their useful correspondence. The authors would also like to thank two anonymous referees for their constructive comments which enabled us to correct some of the results and improve the presentation of the paper.

\section{REFERENCES}

[AJ94] S. Abramsky and A. Jung. Domain theory. in: S. Abramsky, D.M. Gabbay, T.S.E. Maibaum (Eds.). Handbook of Logic in Computer Science, Vol III, Clarendon Press, Oxford (1994).

[AP10] M. Ali-Akbari and M. Pourmahdian. Completeness of hyperspace of compact subsets of quasimetric spaces. Acta Mathematica Hungarica, 127: 260-272, 2010.

[AHP09] M. Ali-Akbari, B. Honari and M. Pourmahdian. Any $T_{1}$ space has a continuous poset model. Topology and its Applications, 156: 2240-2245, 2009.

[AHPR09] M. Ali-Akbari, B. Honari, M. Pourmahdian and M. M. Rezaii. The space of formal balls and models of quasi-metric spaces. Mathematical Structures in Computer Science, 19: 337-355, 2009.

[Ber10] U. Berger, J. Blanck and P.K. Kober. Domain representations of spaces of compact subsets. Mathematical Structures in Computer Science, 20(2): 107-126, 2010.

[Bla97] J. Blanck. Domain representability of metric spaces. Annals of Pure and Applied Logic, 83: 225247, 1997.

[Bla00] J. Blanck. Domain representations of topological spaces. Theoretical Computer Science, 247: 229-255, 2000.

[BBR98] M.M. Bonsangue, F. van Breugel and J.J.M.M. Rutten. Generalized metric spaces: Completion, topology and powerdomains via the Yoneda embedding. Theoretical Computer Science, 193: 1$51,1998$.

[CR06] J. Cao and J. Rodríguez-López. On hyperspace topologies via distance functionals in quasi-metric spaces. Acta Mathematica Hungaria, 112: 249-268, 2006.

[Eda95a] A. Edalat. Dynamical systems, measures and fractals via domain theory. Information and Computation, 120: 32-48, 1995.

[Eda95b] A. Edalat. Domain Theory and Integration. Theoretical Computer Science, 151: 163-193, 1995.

[Eda97] A. Edalat. Domains for computation in mathematics, physics and exact real arithmetic. Bulletin of Symbolic Logic, 3: 401-452, 1997. 
[ES99] A. Edalat and Ph. Sünderhauf. Computable Banach spaces via domain theory. Theoretical Computer Science, 219: 169-184, 1999.

[EH98] A. Edalat and R. Heckmann. A computational model for metric spaces. Theoretical Computer Science, 193: 53-73, 1998.

[FL82] P. Fletcher and W. Lindgren. Quasi-uniform spaces. Marcel Dekker, New York, 1982.

[GRS08] L.M. Garcia-Raffi, S. Romaguera and M.P. Schellekens. Applications of the complexity space to the general probabilistic divide and conquer algorithms. Journal of Mathematical Analysis and Applications, 348: 346-355, 2008.

$\left[\mathrm{GHK}^{+}\right.$03] G.K. Gierz, K.H. Hofmann, K. Keimel, J.D. Lawson, M. Mislove and D.S. Scott. Continuous lattices and domains. Encyclopedia of Mathematics and its Applications, Vol. 93, Cambridge University Press, 2003.

[Hec99] R. Heckmann. Approximation of metric spaces by partial metric spaces. Applied Categorical Structures, 7: 71-83, 1999.

[Hut85] J.E. Hutchinson. Fractals and self-similarity. Indiana University Mathematics Journal, 30: 10591066, 1985.

[Kel63] J.C. Kelly. Bitopological spaces. Proceedings of the London Mathematical Society, 13: 71-89, 1963.

[Kni91] P. Knijnenburg. Algebraic domains, chain completion and the powerdomain construction. Technical report, Utrecht University, 1991.

[KKW04] R. Kopperman, H.-P. A. Künzi and P. Waszkiewicz. Bounded complete models of topological spaces. Topology and its Applications, 139: 285-297, 2004.

[Kün95] H.-P. A. Künzi. Nonsymmetric topology. in Proceedings of Colloquium on topology, Szekszard, Hungary, colloquium Mathematical Society János Bolyai Mathematic Studies, 4: 303-338, 1995.

[Kü02] H.-P. A. Künzi. Quasi-metrizable spaces satisfying certain completeness conditions. Acta Mathematica Hungaria, 95: 345-357, 2002.

[KS02] H.-P. A. Künzi and M.P. Schellekens. On the Yoneda completion of a quasi-metric space. Theoretical Computer Science, 278: 159-194, 2002.

[Law97] J.D. Lawson. Spaces of maximal points. Mathematical Structures in Computer Science, 7: 543$555,1997$.

[Law98] J.D. Lawson. Computation on metric spaces via domain theory. Topology and its Applications, 85: 263-274, 1998.

[LK04] J. Liang and H. Kou. Convex power domain and Vietoris space. Computers and Mathematics with Applications, 47: 541-548, 2004.

[BL08] H. Bennett and D. Lutzer. Domain-representability of certain complete spaces. Houston Journal of Mathematics, 34: 753-772, 2008.

[Mark78] G. Markowski. Chain-complete partial order sets and directed sets with applications. Algebra Universalis, 6: 53-68, 1978.

[Mar98] K. Martin. Domain theoretical models of topological spaces. Electronic Notes in Theoretical Computer Science, 13: 173-181, 1998.

[Mar04] K. Martin. Fractals and domain theory. Mathematical Structures in Computer Science, 14, 833$851,2004$.

[MMR02] K. Martin, M. Mislove and G.M. Reed. Topology and domain theory. Recent Progress in General Topology II, ed. by M. Husek and J. van Mill, Pages 371-394 Elsevier, Amsterdam, 2002.

[RR02] J. Rodríguez-López and S. Romaguera. The relationship between the Vietoris topology and the Hausdorff quasi-uniformity. Topology and its Applications, 124: 451-464, 2002.

[RRV08] J. Rodríguez-López, S. Romaguera and O. Valero. Denotational semantics for programming languages, balanced quasi-metrics and fixed points. International Journal of Computer Mathematics, 85: 623-630, 2008 .

[RSV09] J. Rodríguez-López, M.P. Schellekens and O.Valero. An extension of dual complexity space and an application to computer science. Topology and its Applications, 156: 3052-3061, 2009.

[RSV03] S. Romaguera, E.A. Sánchez-Pérez and O. Valero. Computing complexity distances between algorithms. Mathematical methods in computer science and the information theory, Kybernetika, 39: 569-582, 2003. 
[RS99] S. Romaguera and M.P. Schellekens. Quasi-metric Properties of complexity classes. II Iberoamerican Conference on Topology and its Applications. Topology and its Applications, 98: 311-322, 1999.

[RV08] S. Romaguera and O. Valero. On the structure of the space of complexity partial functions. International Journal of Computer Mathematics, 85: 631-640, 2008.

[RV09] S. Romaguera and O. Valero. A quantitative computational model for complete partial metric spaces via formal balls. Mathematical Structures in Computer Science, 19: 541-563, 2009.

[RV10] S. Romaguera and O. Valero. Domain theoretic characterisations of quasi-metric completeness in terms of formal balls. Mathematical Structures in Computer Science, 20: 453-472, 2010.

[Rut98] J.J.M.M. Rutten. Weighted colimits and formal balls in generalized metric spaces. Topology and its Applications, 89: 179-202, 1998.

[Sch03] M.P. Schellekens. A characterization of partial metrizability: Domains are quantifiable. Theoretical Computer Science, 305: 409-432, 2003.

[Smy83] M.B. Smyth. Power domains and predicate transformers: A topological view. Lecture notes in computer science, 154: 662-675, Springer-Verlag, 1983.

[Was06] P. Waszkiewicz. Partial metrizability of continuous posets. Mathematical Structures in Computer Science, 16: 359-372, 2006.

[Wes57] J.D. Weston. On the comparison of topologies. Journal of the London Mathematical Society, 32: 342-354, 1957. 